\title{
Análisis de las
}

publicaciones

periódicas marciales

chinas editadas durante

el periodo republicano

W illiam Acevedo $\&$ Mel Cheung

Academy Northern Chinese Martial Arts (Canadá)

Recibido 22/11/2010 - Aceptado 15/03/2011

\section{Resumen}

El presente artículo estudia las revistas y periódicos de temática marcial publicados en China durante el periodo republicano (1912-1949). Las fuentes principales del estudio han sido el índice y los treinta volúmenes de la Colección de Periódicos de Artes Nacionales del Periodo Republicano (Mingguo Guoshu Qi Kan Wenxian Jicheng), editados por Shi Yong Xing en el año 2008. Además de diversos datos básicos referentes a cada una de las 45 publicaciones localizadas (título, editor, lugar de publicación y permanencia), el trabajo hace hincapié en el contexto en que se produce esta eclosión de publicaciones marciales, caracterizado por los intentos de China por surgir como una nación digna de ser reconocida en el teatro mundial. A modo de ejemplo de la riqueza de informaciones contenidas en estas revistas y periódicos, se analizan diversos aspectos específicos sobre las artes marciales del periodo, desde su propia definición hasta su contribución a la exaltación del espíritu nacionalista chino, a la preparación del ejército y en general al fortalecimiento de la nación.

Palabras clave: Artes marciales chinas, guoshu, Mingguo, Jingwu, Esencia Marcial, Academia Central de Artes Nacionales.

Analysis of the Chinese martial periodicals edited during the Republican Era Abstract: The present article studies the periodicals and magazines published during the Chinese Republican period (1912-1949). The main sources for this work are the index and thirty volumes of the Republican Era Collection of National Arts Periodicals (Mingguo Guoshu Qi Kan Wenxian Jicheng), edited by Shi Yong Xing in 2008. Besides basic data about the 45 publications included in the collection such as title, editor, publication information and period of existence; this article emphasises the historical context in which these publications appeared as well as their efforts to help China becoming a nation recognized at the world stage. As an example of the wealth of information contained in these periodicals regarding Chinese martial arts, we have analyzed the terminology that 
PORTADA DE UNO DE LOS NÚMEROS DE LA REVISTA

ESENCIA MARCIAL, PUBLICADO EN 1923.

Todas las fotografías son cortesía de W illiam Acevedo y M ei Cheung. was used to define them, the publications' contribution to exalt nationalism up to their influence in strengthening of China's citizens and military.

Key words: Chinese martial arts, guoshu, Mingguo, Jingwu, Martial Essence, Central National Arts Academy.

\section{Análise de publicações periódicas marciais chinesas editadas durante o período republicano}

Resumo: 0 presente artigo estuda as revistas e os periódicos de temática marcial publicadas na China durante o período republicano (1912-1949). A fonte principal do estudo foi 0 índice de trinta volumes da Colecção de Periódicos das Artes Nacionais do Período Republicano (M ingguo Guoshu Qi Kan Wenxian Jicheng), editados por Shi

Yong Xing, no ano de 2008. Para além de diversos dados básicos referentes a cada uma das 45 publicações encontradas (título, editor, local de publicação e periodicidade), o trabalho enfatiza o contexto em que se produz essa eclosão de publicações marciais, caracterizado pelos intentos da China surgir como uma nação digna de ser reconhecida no palco mundial. A título de exemplo, procura-se analisar a riqueza de informações contidas nestas revistas e periódicos, os diversos aspectos específicos sobre as artes marciais desse período, desde a sua própria definição até a sua contribuição para a exaltação do espírito nacionalista chinês, bem como a preparação dos exércitos e, em geral, do fortalecimento da nação.

Palavras-chave: Artes marciais chinesas, guoshu, Mingguo, Jingwu, essência M arcial, A cademia Central de Artes Nacionais. 


\section{A nálisis de las}

publicaciones

periódicas marciales

chinas editadas durante

el periodo republicano

W illiam Acevedo $\&$ Mel Cheung

Academy Northern Chinese Martial Arts (Canadá)

\section{Introducción}

Las derrotas sufridas por China en las Guerras del O pio (1839-1842 y 18561860), Rebelión Taiping (1850-1864), primera guerra Chino-Japonesa (1894-1895) y Rebelión de los Bóxeres (1898-1901), así como las concesiones realizadas a la Alianza de las $\mathrm{O}$ cho $\mathrm{N}$ aciones tras esta última, sirvieron para consolidar la imagen del pueblo chino como los hombres enfermos de Asia. Buscando fortalecer a la nación y erradicar esta imagen, en 1915, ya durante la República China (1912-1949), aparecieron dos diferentes ideologías, el Nuevo M ovimiento Cultural (Xin W enhua Yundong) y la Escuela de Esencia Nacional (Guocui). El Nuevo Movimiento Cultural propuso el rechazo de los ideales confucianos y la adopción de sistemas occidentales en diversos campos, incluyendo el de la educación física. En contraste, la Escuela de Esencia Nacional buscó la implementación de modelos autóctonos, hasta el extremo de llegar a afirmar que los sistemas de educación física occidentales no tendrían ningún efecto sobre los cuerpos chinos (Brownell, 1995)².

\footnotetext{
1 El Confucianismo en sus orígenes consideraba la práctica física/marcial y los estudios de tipo académico como elementos complementarios en la formación del estudiante (Acevedo, Gutiérrez $\&$ Cheung, 2010). Con el paso del tiempo los eruditos confucianos comenzaron a enfatizar los estudios académicos y a relegar los estudios marciales. Una de las escuelas con mayor influencia en esta tendencia fue la escuela neo-confucianista de Li, creada por Zhu Xi (1130-1200 d.C.), la cual primaba la no acción sobre la acción y consideraba la ejercitación de los cuerpos como una desviación (Ma, 2009). Durante el s. XIX, numerosas referencias publicadas por cronistas occidentales y manchúes degradaban la práctica marcial en China al ámbito de lo inútil (véase e.g. Couch, 2009). Esto podría deberse en parte a las observaciones de técnicas o practicantes cuyas habilidades no estaban enfocadas a la eficacia en el combate sino a la exhibición, así como también a la xenofobia que sufría un pueblo considerado como débil. Estas antiguas actitudes que desdeñaban la práctica marcial/física en beneficio de la intelectual aún eran predominantes a principios del siglo XX, cuando China vivía momentos críticos.
} 
A pesar de la resistencia del Nuevo Movimiento Cultural, y en un esfuerzo de síntesis, China buscó la adaptación de aquellos elementos foráneos que le ayudarían a modernizar sus fuerzas armadas y también a fortalecer a sus ciudadanos, y al mismo tiempo preservar su identidad cultural.

En el ámbito específico de las artes marciales, se persiguió la unión de las diferentes escuelas y practicantes, así como mejorar la calidad de las artes de lucha. Entre las diversas iniciativas desarrolladas se realizó un gran esfuerzo editorial, publicándose durante este periodo cientos de manuales de entrenamiento y periódicos/revistas (qi kan) marciales. Es a estos últimos a los que dedicaremos nuestra atención en las siguientes páginas.

\section{Método}

El presente artículo está basado en el análisis de la Colección de Periódicos de Artes Nacionales del Periodo Republicano (M ingguo Guoshu Qi Kan W enxian Jicheng) editada por Shi Yong Xing (2008). Esta colección está conformada por un índice y treinta volúmenes que recopilan las publicaciones periódicas de artes marciales y deportes que aún se encuentran en bibliotecas como la del Departamento de Deportes de la Universidad de Zhejiang, Biblioteca Nacional, Biblioteca de Shanghái, Biblioteca de la Universidad de Beijing, Biblioteca de la Universidad de Xiamen, colecciones privadas, etc. En ella se incluyen las ediciones de las publicaciones republicanas que sobrevivieron a la segunda guerra mundial y a la revolución cultural; sin embargo, no todos los números publicados se salvaron 0 se encontraron a tiempo para ser incluidos en la colección. Este proyecto de recopilación contó con un equipo de trabajo que dedicó dos años a su ejecución.

Las publicaciones periódicas incluidas en la colección ${ }^{2}$ se publicaron en las provincias o ciudades del este de China; el 35,56\% tuvo su centro en la ciudad de Shanghái, 13,33\% en la provincia de Jiangsu, 11,11\% en la provincia de Shandong, $8,89 \%$ en la provincia de Zhejiang, 4,44\% (cada una) en la provincia de Shanxi y en las ciudades de Beijing, Tianjin, la isla de Hong Kong y otros centros que no fue posible identificar, y 2,22\% cada una en las provincias de Guangdong, Fujian, Hunan y en la ciudad de Tokio. Como se puede apreciar, ninguna de estas publicaciones se editó en las provincias del oeste como son Gansu, Guizhou, Q inghai, Shaanxi, Sichuan o Yunnan, así como en las regiones autónomas, aunque puede que sí fuesen distribuidas de manera limitada en estas y otras provincias. Este hecho limitó la cobertura de los periódicos marciales, debido probablemente a la falta de control político que el partido nacionalista tenía sobre la nación en el periodo republicano. Algunas de las publicaciones se trasladaron fuera de su lugar de edición original durante la agresión japonesa (1931-1945); sin embargo, la toma del poder por parte de los comunistas en 1949 terminó con ellas definitivamente.

Es importante señalar que el método usado en la colección para la reproducción de estas publicaciones (fotocopia de los originales) no permite que en ocasiones las fotografías, y en algunos casos los textos originales, sean legibles 0 reproducibles. Debido al gran volumen de revistas y artículos publicados, nos

2 Algunas publicaciones periódicas cambiaron su nombre en varias ocasiones. En este recuento cada título ha sido contabilizado como una nueva publicación. 
hemos limitado a utilizar las introducciones generales que se hacen de cada publicación en el índice y solo hemos verificado su exactitud en la publicación original correspondiente; en casos puntuales también se han utilizado artículos específicos de las revistas.

\section{Promoviendo las artes marciales a través de la pluma}

Durante el periodo 1921-1949 la Colección de Periódicos de Artes Nacionales del Periodo Republicano recoge un total de 45 publicaciones periódicas en las zonas citadas (véase Tabla I). Estas publicaciones periódicas fueron las primeras en las que se discutió de manera abierta diversos temas relacionados con las artes marciales y la cultura física.

Tabla 1. Periódicos y revistas marciales publicados en China durante la República China (1912-1949).

\begin{tabular}{|c|c|c|c|}
\hline Titulo & $\begin{array}{l}\text { Ciudad } \\
\text { Provincia }\end{array}$ & Periodo & Organización \\
\hline Artes Marciales & Shanghái & 1921-? & Organización de Caballeros Marciales \\
\hline $\begin{array}{l}\text { Artes Nacionales: Revista } \\
\text { M ensual de la A cademia } \\
\text { de Artes Nacionales de la } \\
\text { Provincia de Zhejiang }\end{array}$ & Zhejiang & 1929 & $\begin{array}{l}\text { Academia de Artes Nacionales de } \\
\text { Zhejiang }\end{array}$ \\
\hline $\begin{array}{l}\text { A sociación China de Artes } \\
\text { Marciales de Shanghái }\end{array}$ & Shanghái & 1921-? & $\begin{array}{l}\text { A sociación China de Artes Marciales } \\
\text { de Shanghái }\end{array}$ \\
\hline $\begin{array}{l}\text { Asociación Esencia Marcial } \\
\text { Edición Especial }\end{array}$ & Shanghái & 1923-? & $\begin{array}{l}\text { Asociación Atlética de la Esencia } \\
\text { Marcial de Shanghái }\end{array}$ \\
\hline Central & Shanghái & $\begin{array}{c}1922 ?- \\
23 ?\end{array}$ & $\begin{array}{l}\text { Asociación Atlética de la Esencia } \\
\text { Marcial de Shanghái }\end{array}$ \\
\hline $\begin{array}{l}\text { Colección de Discursos de Artes } \\
\text { Nacionales del Señor Zhang } \\
\text { Zhi Jiang }\end{array}$ & $?$ & $?$ & 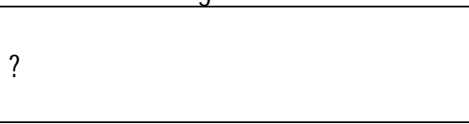 \\
\hline Deportes & Beijing & 1932 & Escuela de Artes Nacionales de Beijing \\
\hline $\begin{array}{l}\text { Edición Especial de Artes } \\
\text { Nacionales del Condado de Yin }\end{array}$ & $\begin{array}{l}\text { Condado de Yin } \\
\text { (Zhejiang) }\end{array}$ & 1931 & $\begin{array}{l}\text { Asociación para el Estudios de } \\
\text { Deportes Nacionales }\end{array}$ \\
\hline $\begin{array}{l}\text { Edición Especial de la } \\
\text { Conferencia Recreativa de Artes } \\
\text { Nacionales de Zhejiang }\end{array}$ & Zhejiang & 1929 & Academia Central de Artes Nacionales \\
\hline $\begin{array}{l}\text { Edición Especial del Movimiento } \\
\text { de Artes Nacionales }\end{array}$ & Zhejiang & 1936 & $\begin{array}{l}\text { Escuela de Artes Nacionales de la } \\
\text { Provincia de Zhejiang }\end{array}$ \\
\hline $\begin{array}{l}\text { Edición Especial del Primer } \\
\text { Examen Nacional }\end{array}$ & $\begin{array}{l}\text { Nanjing } \\
\text { (Jiangsu) }\end{array}$ & 1928 & A cademia Central de Artes Nacionales \\
\hline $\begin{array}{l}\text { Edición Especial del Segundo } \\
\text { Examen Nacional de Artes } \\
\text { Nacionales }\end{array}$ & $\begin{array}{l}\text { Nanjing } \\
\text { (Jiangsu) }\end{array}$ & 1933 & Academia Central de Artes Nacionales \\
\hline Esencia Marcial & Shanghái & $1924 ?-39$ & $\begin{array}{l}\text { Asociación Atlética de la Esencia } \\
\text { Marcial de Shanghái }\end{array}$ \\
\hline Esencia Marcial de Xiamen & Xiamen (Fujian) & 1934 & $\begin{array}{l}\text { Departamento Académico Deportivo } \\
\text { de la Esencia Marcial de Xiamen }\end{array}$ \\
\hline Esencia Marcial Pictórica & Shanghái & $1927-32$ & Asociación Central de Esencia Marcial \\
\hline Espíritu Heroico & Shandong & 1936 & $\begin{array}{l}\text { Sociedad Experimental de Salud de } \\
\text { Jinan }\end{array}$ \\
\hline Estudios M arciales & Tokio & 1909 & Liga Unida China \\
\hline $\begin{array}{l}\text { Las Ultimas Reglas de Artes } \\
\text { Nacionales }\end{array}$ & Shanghái & 1933 & Editorial Deportiva de Shanghái \\
\hline
\end{tabular}




\begin{tabular}{|c|c|c|c|}
\hline Titulo & $\begin{array}{l}\text { Ciudad } \\
\text { Provincia }\end{array}$ & Periodo & Organización \\
\hline $\begin{array}{l}\text { Libro de Aniversario de la } \\
\text { Esencia Marcial }\end{array}$ & Shanghái & 1919 & $\begin{array}{l}\text { Asociación Atlética de la Esencia } \\
\text { Marcial de Shanghái }\end{array}$ \\
\hline $\begin{array}{l}\text { M ejoramiento de las } \\
\text { Habilidades de A taque }\end{array}$ & Shandong & $1936 ?$ & $\begin{array}{l}\text { Sociedad Experimental de Salud de } \\
\text { Jinan }\end{array}$ \\
\hline $\begin{array}{l}\text { Periódico Mensual de la Esencia } \\
\text { Marcial de Foshan }\end{array}$ & $\begin{array}{c}\text { Foshan } \\
\text { (Guangdong) }\end{array}$ & $1925-28$ & Esencia Marcial de Foshan \\
\hline $\begin{array}{l}\text { Primavera y Otoño de la Esencia } \\
\text { Marcial }\end{array}$ & Shanghai & 1929-? & $\begin{array}{l}\text { Asociación Central de la Esencia } \\
\text { Marcial de Shanghái }\end{array}$ \\
\hline $\begin{array}{l}\text { Publicación M ensual de } \\
\text { Búsqueda de la Verdad }\end{array}$ & Shandong & 1935 & $\begin{array}{l}\text { Sociedad Experimental de Salud de } \\
\text { Jinan }\end{array}$ \\
\hline $\begin{array}{l}\text { Publicación Trimestral de la } \\
\text { Búsqueda de la Verdad }\end{array}$ & Shandong & 1934 & $\begin{array}{l}\text { Sociedad Experimental de Salud de } \\
\text { Jinan }\end{array}$ \\
\hline Registro de Artes Nacionales & Shandong & 1936-? & $\begin{array}{l}\text { Sociedad Experimental de Salud de } \\
\text { Jinan }\end{array}$ \\
\hline Reglas de Artes Nacionales & Shanghái & 1948 & Estadio Deportivo Jiang de Shanghái \\
\hline Reglas de Artes Nacionales & $?$ & 1936 & $\begin{array}{l}\text { Asociación China de Deportes } \\
\text { Nacionales }\end{array}$ \\
\hline $\begin{array}{l}\text { Reporte de la A sociación de } \\
\text { Esencia Marcial }\end{array}$ & Shanghái & 1933-47 & $\begin{array}{l}\text { Asociación Atlética de la Esencia } \\
\text { Marcial de Shanghái }\end{array}$ \\
\hline $\begin{array}{l}\text { Revista Anual de Artes } \\
\text { Nacionales de la Provincia de } \\
\text { Jiangsu }\end{array}$ & Jiangsu & 1929 & $\begin{array}{l}\text { Academia de Artes Nacionales de la } \\
\text { Provincia de Jiangsu }\end{array}$ \\
\hline $\begin{array}{l}\text { Revista Anual de la Esencia } \\
\text { Marcial }\end{array}$ & Shanghai & 1931 & $\begin{array}{l}\text { Asociación Atlética de la Esencia } \\
\text { Marcial de Shanghái }\end{array}$ \\
\hline $\begin{array}{l}\text { Revista Central de Artes } \\
\text { Nacionales }\end{array}$ & $\begin{array}{l}\text { Nanjing } \\
\text { (Jiangsu) }\end{array}$ & 1929 & Academia Central de Artes Nacionales \\
\hline Revista de Deportes & Beijing & $1912-?$ & $\begin{array}{l}\text { Centro de Investigación Deportiva de } \\
\text { Beijing }\end{array}$ \\
\hline $\begin{array}{l}\text { Revista de Deportes y Artes } \\
\text { Nacionales de Shanxi }\end{array}$ & Shanxi & 1934-35 & $\begin{array}{l}\text { Asociación para la Promoción de las } \\
\text { Artes Nacionales de la Provincia de } \\
\text { Shanxi }\end{array}$ \\
\hline $\begin{array}{l}\text { Revista de la Academia Central } \\
\text { de Artes Nacionales }\end{array}$ & $\begin{array}{c}\text { Nanjing } \\
\text { (Jiangsu) } \\
\end{array}$ & 1928 & Academia Central de Artes Nacionales \\
\hline Revista de la Esencia Marcial & Hong Kong & 1925-? & Asociación Esencia Marcial Atlética \\
\hline Revista Esencia Marcial & Shanghái & $\begin{array}{c}1923 ?- \\
24 ?\end{array}$ & $\begin{array}{l}\text { Asociación Atlética de la Esencia } \\
\text { Marcial de Shanghái }\end{array}$ \\
\hline $\begin{array}{l}\text { Revista M ensual de Artes } \\
\text { Nacionales de Shanxi }\end{array}$ & Shanxi & 1939 & $\begin{array}{l}\text { Centro de Investigación de Artes } \\
\text { Nacionales Condado de Cheng } \\
\text { Provincia de Shanxi }\end{array}$ \\
\hline $\begin{array}{l}\text { Revista M ensual de Artes } \\
\text { Nacionales Unidas }\end{array}$ & Shanghái & $1934-35$ & $\begin{array}{l}\text { Sociedad de la Revista M ensual de } \\
\text { Artes Nacionales Unidas de Shanghái }\end{array}$ \\
\hline $\begin{array}{l}\text { Revista Quincenal de Artes } \\
\text { Nacionales de la provincia de } \\
\text { Hunan }\end{array}$ & Hunan & 1932 & $\begin{array}{l}\text { Centro de Entrenamiento de Artes } \\
\text { Nacionales de Hunan }\end{array}$ \\
\hline $\begin{array}{l}\text { Revista Quincenal de Artes } \\
\text { Nacionales de Shanghái }\end{array}$ & Shanghai & 1932 & $\begin{array}{l}\text { Sociedad de Propagación Marcial y de } \\
\text { las Artes Nacionales de Shanghái }\end{array}$ \\
\hline $\begin{array}{l}\text { Revista Semanal de Artes } \\
\text { Nacionales }\end{array}$ & Tianjin & 1935 & $\begin{array}{l}\text { Sociedad Moral de Estudios Marciales } \\
\text { de Tianjin }\end{array}$ \\
\hline $\begin{array}{l}\text { Revista Semanal de Artes } \\
\text { Nacionales }\end{array}$ & $\begin{array}{l}\text { Nanjing } \\
\text { (Jiangsu) }\end{array}$ & 1930-? & Casa Editorial de Nanjing \\
\hline Salud y Belleza ${ }^{3}$ & Hong Kong & 1947 & $\begin{array}{l}\text { Escuela de Deportes de Li en Hong } \\
\text { Kong }\end{array}$ \\
\hline $\begin{array}{l}\text { Volumen M ensual de Artes } \\
\text { Nacionales }\end{array}$ & Tianjin & 1934-? & $\begin{array}{l}\text { Academia de Artes Nacionales de } \\
\text { Tianjin }\end{array}$ \\
\hline Voz del Arte Nacional & Shanghái & $\begin{array}{l}1928-32 \\
1935-36 \\
\end{array}$ & $\begin{array}{l}\text { Escuela de Artes Nacionales de la } \\
\text { Municipalidad de Shanghái }\end{array}$ \\
\hline
\end{tabular}


Las dos organizaciones más importantes, y cuyos periódicos se publicaron durante la mayor parte del periodo republicano, fueron la Asociación Atlética de la Esencia Marcial de Shanghái (Shanghai Jingwu Tiyu Zong Hui), siendo una iniciativa de tipo privado, y la Academia Central de Artes Nacionales de Nanjing (Nanjing Zhongyang Guoshu Guan), patrocinada por el gobierno nacionalista. A pesar de que la gran mayoría del esfuerzo editorial se debe a estas dos organizaciones, no fueron las únicas. Otros grupos también editaron sus propios periódicos o revistas, aunque habitualmente con mayores dificultades ${ }^{4}$.

La falta de financiación para este tipo de publicaciones hizo que por primera vez se publicitasen productos de consumo como la coca-cola o la conocida pomada del tigre. Otro obstáculo que debieron afrontar los promotores de estas publicaciones fue la agresión japonesa sobre la ciudad de Shanghái en 1932 (como hemos señalado, en esta ciudad se editó el mayor porcentaje de publicaciones periódicas marciales), la cual dañó severamente la ciudad. Este hecho fue reconocido por Zheng Tian Shi, editor de la revista Reporte de la Asociación de Esencia Marcial (Jingwu Cong Bao, 1933-1947), quien señalaba:

La guerra comenzó el 28 de enero y fue suficientemente brutal para destruir muchas cosas. Espero que de ahora en adelante el espíritu de la Esencia Marcial ayude a que nuestra nación se sobreponga. Los colegas que trabajan con la Esencia M arcial no perderán tiempo para hacer todo lo posible para mejorar nuestros deportes [...] este periódico cubre las actividades que estamos realizando para el bienestar de nuestra sociedad y para recuperar el espíritu de los deportes.

(en Shi, 2008, 21: 1).

Como puede apreciarse, este párrafo revela el compromiso de la asociación para lograr la recuperación nacional, compromiso al que no serían ajenas un gran número de asociaciones e instituciones.

Muchas de las publicaciones del periodo fueron ediciones especiales, en algunos casos debido a la falta de fondos para mantener constante su periodicidad y en otros porque ni siquiera existían planes para continuar la publicación de manera periódica. Esta situación limitó la cobertura y el alcance de estas revistas, situación que no escapó de las críticas, como la que realiza Liu Fu Min, editor de la Revista Quincenal de Artes Nacionales de Shanghái (Guoshu Ban Yue Kan Shanghai, 1932):

Caballeros, recientemente se han publicado muchas clases de revistas, esto es genial, las artes marciales pueden ser descritas en palabras; para revelar su verdadero significado, se debe hacer a través de revistas.

\footnotetext{
${ }^{3}$ Esta publicación trata de deportes occidentales como el boxeo; a pesar de no ser una revista de artes marciales fue incluida en la colección, ya que el boxeo fue estudiado y analizado por los proponentes del guoshu.

4 Como ejemplo, en 1921 la Asociación China de Artes Marciales de Shanghái (Shanghai Zhong Hua W ushu Hui, 1921-?) (edición especial dedicada a la 3a Reunión de Miembros) publicaba: "LoS fondos de la asociación se basan en las contribuciones de sus miembros, si alguna circunstancia no planeada ocurre, no podremos cubrirla. Por este motivo solicitamos la generosa colaboración de nuestros miembros" (en Shi, 2008, 7: 377).
}

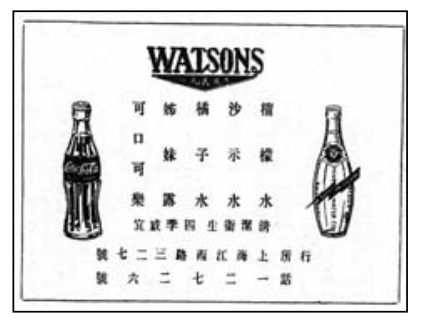

Publicidad de Coca Cola y Agua Mineral en el PERIÓDICO DE LA ESENCIA Marcial, 1933. 
Esto atraerá la atención de la gente y la nación hacia las artes marciales. Por lo tanto esta revista busca simplificar el entendimiento hacia el guoshu para hacerlo popular y mejorar su futuro desarrollo. Sin embargo es muy desafortunado que existan pocas revistas con un contenido y publicación constantes. Estas son las debilidades más grandes si queremos promover una nación y una sociedad fuertes.

(en Shi, 2008, 14: 4).

\section{Instando al fortalecimiento de la nación}

Un tema recurrente en las diferentes introducciones y artículos escritos durante este periodo es el de fortalecer la nación a través de la cultura física. Esta tesis la explica Mao Zedong (1893-1976), quien se convertiría en el líder del partido comunista, en un artículo publicado en 1917:

[...] Confucio tomó las enseñanzas del arquero y del conductor de carros. En Alemania, la educación física ha alcanzado la mayor popularidad. La esgrima se ha expandido en toda la nación. Japón tiene el bushido. Además, recientemente, siguiendo la tradición de nuestro país, el judo se ha desarrollado allí a un nivel admirable

[...] La razón principal de la educación física es el heroísmo militar. Los elementos que lo componen, como coraje, audacia, intrepidez y perseverancia, son todos una cuestión de voluntad.

(Mao, 1917).

Los proponentes de la Escuela de Esencia Nacional manifestaron ideas similares en sus escritos. Un ejemplo se encuentra en la revista Artes Marciales (W ushu, 1921-?) publicada en 1921 por la Organización de Artes Marciales Chinas, la cual fue bautizada en sus inicios como Organización de Caballeros Marciales. En su primera edición su editor Xie Qiang Gon, escribía:

Existen muchos caminos para salvar a nuestra nación, yo creo que la única forma para alcanzar este objetivo es a través de la rápida promoción de los deportes. Hoy en día estas prácticas han alcanzado su punto más alto, sin embargo en nuestro país es lo opuesto. Considero que hay una solución, que es la promoción de los deportes nacionales. Shanghái es nuestro centro y desde aquí nos hemos expandido a otras áreas. ¿Por qué no extendemos este fuego? ¿Por qué no desarrollamos nuestra nueva nación y su gente a través de los deportes? Q ueremos que esta revista sea el principio de este proceso, como un medio para atraer a aquellos con experiencia en nuestros deportes nacionales quienes con su coraje y determinación lograrán alcanzar esta meta.

(en Shi, 2008, 1: 327).

Desde diversas organizaciones gubernamentales, como la Academia de Artes Nacionales de Zhejiang también se promovió este ideal, como se señala en Artes Nacionales: Revista Mensual de la Academia de Artes Nacionales de la Provincia de Zhejiang (Guoshu: Zhejiang Sheng Guoshu Guan Yue Kan, 1929):

Es my difícil encontrar un ciudadano chino en forma, con el afán de aprender cosas nuevas, con una fuerte y adecuada actitud en la vida, a 
estos les hace falta coraje y motivación. Ellos tienen las características de los hombres enfermos; ¿cuántas generaciones deberán pasar antes de que nos demos cuenta de que esta situación debe cambiar? Debemos despertar a esta realidad. Las Artes $\mathrm{N}$ acionales son el remedio contra la enfermedad de los hombres enfermos de Asia del este, esta es la mejor arma para fortalecer el cuerpo y proteger la nación.

(en Shi, 2008, 10: 11).

El párrafo anterior ilustra claramente el sentido autocrítico de aquellos que proponían un movimiento de renovación y su afán por cambiar la situación en la que se encontraba el país. En la siguiente cita, extraída de un artículo en inglés, publicado en 1929 en Artes Nacionales: Revista M ensual de la Academia de Artes Nacionales de la Provincia de Zhejiang con el título "El Renacimiento del Arte Atlético Chino" ("The Renaissance of the Chinese Athletic Art"), se sintetizan los objetivos que impulsaron este interés en las artes marciales:

Hablando brevemente, los propósitos del gobierno para la promoción de este arte del boxeo chino son:

1. Ayudar a la autodefensa de los ciudadanos a través de la práctica de este arte.

2. Hacer de China una nación poderosa a través de ciudadanos moral y físicamente fuertes.

3. Mantener la paz de manera permanente en los diferentes estamentos al hacer de la China enferma una nación robusta.

(en Shi, 2008, 10: 45-49).

\section{Definiendo las artes marciales}

Durante el periodo republicano se buscó no solo promover la práctica marcial, sino también su estudio técnico y teórico. Las artes marciales chinas han sido descritas de diferentes maneras a lo largo de los siglos, sin embargo no sería hasta principios del S. XX cuando se buscó por primera vez unificarlas mediante un solo término, en este caso guoshu (arte nacional). Previamente a la creación de la Academia Central de Artes Nacionales (Zhongyang Guoshu Guan), se utilizaron diferentes denominaciones como se describe a continuación.

Uno de los primeros programas de entrenamiento para uso a nivel nacional fue publicado en 1911 por Ma Liang (1878-1947) también conocido como Ma Zizhen, un comandante al servicio de uno de los señores de la guerra (líderes militares que lucharon por el control de la nación en el periodo 1916-1928). Ma organizó un currículo que constaba de cuatro volúmenes, a saber: estudio de boxeo chino, estudio de lucha china, estudio de espada de doble filo y estudio de bastón largo. El nombre con el que se bautizó este currículo fue Nuevas Artes Marciales de China (Zhong hua xin wushu). Este programa desaparecería del ámbito nacional en un tiempo muy corto 5 .

5 Aunque el programa de $\mathrm{Ma}$ antecede a las publicaciones periódicas que son el tema de este articulo, lo hemos señalado para dar una idea de los conceptos que se utilizaban para definir a las artes marciales chinas antes de la aparición de estas primeras publicaciones periódicas. 
ALgunOS DE LOS

FUNDADORES DE LA

Academia Central de

Artes Nacionales. De

ARRIBA A ABAJO Y DE DERECHA A IZQUIERDA: CHEN

Diao Yuen, Li De Lin, He Jin Zhi, Li Fang Chen

(LI JING LIN), ZHANG ZHI JIANG, en LA Revista de la Academia Central de Artes Nacionales, 1928.

En 1919, para conmemorar el aniversario de la Esencia Marcial (Jingwu), se publicó una edición especial de sus actividades, incluyendo un artículo en inglés titulado "Historia del Kung Fu Chino" ("History of Chinese Kung Fu"), usando el término que aún hoy en día describe popularmente a las artes marciales chinas. En 1921 se publicaría el periódico Artes Marciales (W ushu) por la O rganización de Caballeros Marciales, la cual, como se puede leer, utilizó el mismo término que $\mathrm{Ma}$ para nombrar su currículo de entrenamiento. Un año más tarde, la A sociación Atlética de la Esencia Marcial de Shanghái comenzaría a publicar el periódico Central (Zhongyang), donde se describen los elementos que integraban su currículo. No se utilizó el término wushu; en su lugar se utilizó una terminología diferente: el departamento de habilidades de ataque (ji ji) divido en las secciones de boxeo chino (quan shu), armas (bing qi), práctica con oponente (dui shou) y trabajo interno (neigong). Otras ramas de la asociación siguieron una estructura similar.

Otra publicación que también utilizó el término de "habilidades de ataque" fue el periódico Mejoramiento de las Habilidades de Ataque (Ji Ji Gai Jing). Este periódico se inició con el nombre de Publicación Trimestral de la Búsqueda de la Verdad (Qiu Shi Ji Kan) en 1934, cambiando su nombre a Publicación Mensual de Búsqueda de la Verdad (Qiu Shi Yue Kan) en 1935 y posteriormente se bautizó en septiembre de 1936 como Espíritu Heroico (Xia Hun) cambiándose su nombre una vez más a Mejoramiento de las Habilidades de A taque.

En la Revista de la Academia Central de Artes Nacionales, publicada en 1928, se utilizaron otros conceptos para describir las artes marciales, como se lee a continuación:

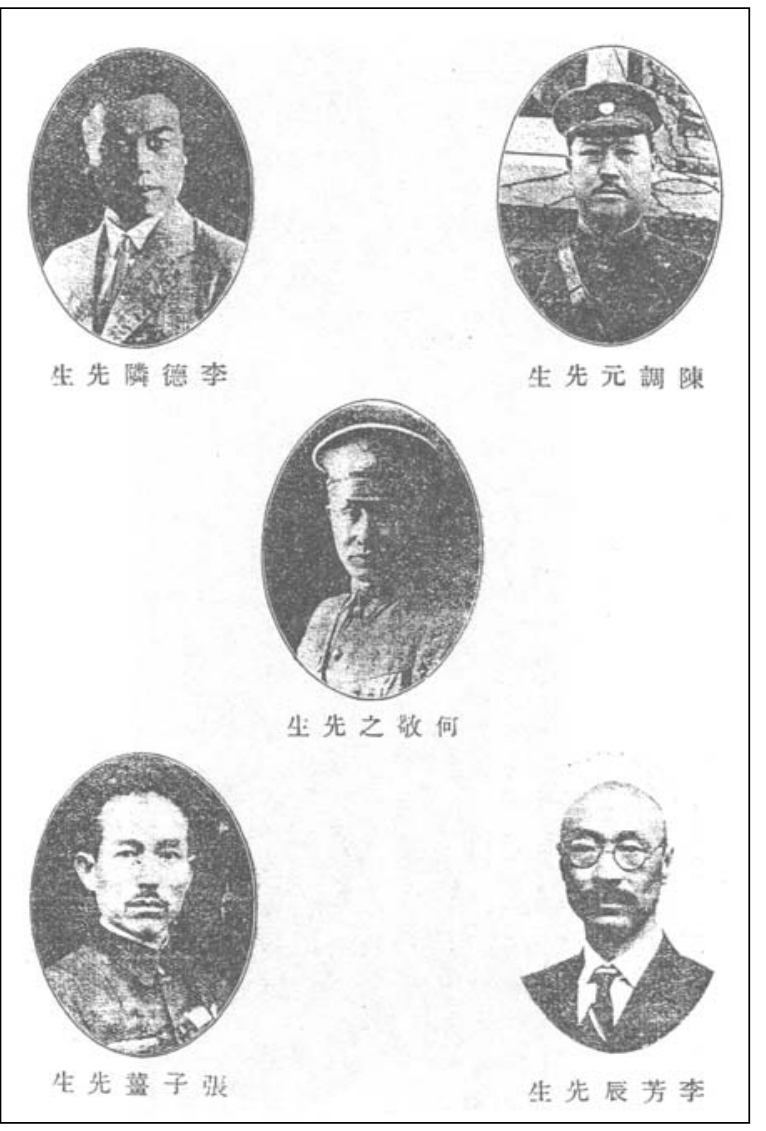

Los elementos que conforman las Artes $\mathrm{Na}$ cionales incluyen el valor (quanyong), las habilidades de ataque (ji ji) y las diferentes habilidades militares (wu yi) de nuestra nación. Las habilidades militares de la nación se han desarrollado desde tiempos antiguos y transmitido a través de muchas generaciones de discípulos; últimamente ha disminuido pero aun existen buenos artistas marciales que usan diferentes métodos para promover estas prácticas, es con la ayuda de los estudios nacionales (guoxue) como se ayudará a su desarrollo.

(en Shi, 2008, 8, 385).

Como se puede apreciar en los anteriores ejemplos, aún bajo la influencia de la Academia Central de Artes Nacionales se continuaron utilizando diferentes términos para referirse a las artes marciales chinas; sin embargo, la Academia impulso el uso del término artes nacionales (guoshu) por razones muy específicas que discutiremos en la siguiente sección.

\section{El concepto de guoshu}

El periodo republicano fue testigo de una ola nacionalista en la que se buscó la preservación de elementos 
culturales autóctonos, entre ellos las artes marciales a las que se bautizó como artes nacionales (guoshu). Este término ha sido considerado en algunos trabajos como una forma simplificada para referirse a las artes marciales chinas (Zhongguo Wushu) (Morris, 2004; An, 2010). Sin embargo, el concepto de guoshu es más amplio, como lo evidencia la introducción de la revista Esencia Marcial (Jingwu), publicada en 1934 por el Departamento Académico Deportivo de la Esencia Marcial de Xia Men, que era una rama de la Asociación Central de la Esencia Marcial de Shanghái. En esta introducción el editor de la revista, Zhuang Nai Gang, explica:

[...] la palabra deporte, proviene de Occidente. Por lo tanto los extranjeros creen que a los chinos no les importa la práctica deportiva y que muchos de nosotros somos como los "hombres enfermos de Asia". Esta podría ser una de las razones por las que nos hemos convertido en este "hombre enfermo" a los ojos de Occidente. Muchos de nosotros no entendemos los beneficios del entrenamiento deportivo, lo cual ha causado que no podamos defendernos frente a los invasores extranjeros; nuestros cuerpos muestran la falta de práctica física, todo lo mencionado es cierto, no podemos negarlo. Sin embargo, no es cierto que a los chinos no nos importen los "deportes". Nosotros tenemos guoshu, el cual no es un solo deporte, sino que cubre muchas otras áreas del entrenamiento físico, lo cual es más amplio que "deporte". "Deporte" solo es el acondicionamiento físico, pero guoshu incluye mantener el estado físico, la salud, la defensa de la nación e incrementar el poder de la nación para las futuras generaciones.

(en Shi, 2008, 22: 258).

En la Edición Especial del Primer Examen Nacional (Di Yi Ci Guokao Te Kan), publicado posteriormente al examen nacional que se realizó el 15 de octubre de 1928 por la Academia Central de Artes Nacionales en N anjing, se comenta que los eventos que se incluyeron fueron pelea a mano vacía, lucha china, pelea con armas largas y cortas. Los candidatos a participar en estas pruebas debieron pasar por un proceso de selección en el que tuvieron que demostrar sus habilidades en rutinas con y sin armas. En el examen nacional, las rutinas a mano vacía y con armas no eran consideradas tan importantes como los eventos de pelea. En la Edición Especial del Segundo Examen Nacional Anual de Artes Nacionales (Zhongyang Di Er Jie Guoshu Guokao Zhuan Kan) se comenta que se eliminaron las pruebas de rutinas a mano libre y con armas. "Artes nacionales" también incluían otras actividades físicas autóctonas como eran el tiro con arco, tiro de balines, juego del gallito, etc. (Ma, 2009). Todo lo anterior muestra claramente que wushu y deporte no incluían todos los elementos que trataba de abarcar el concepto de guoshu.

Adicionalmente, los proponentes de las artes nacionales buscaron la unión de las diferentes escuelas de artes marciales, como lo evidencia la introducción de Artes Nacionales: Revista Mensual de la Academia de Artes Nacionales de la Provincia de Zhejiang, publicada en 1929, al colocar bajo la misma bandera a todos los estilos existentes y donde la única afiliación digna de seguir era la de la nación y por ende la del partido nacionalista.

Adicionalmente, la transmisión de las técnicas ancestrales se ha limitado por el secreto y la proliferación de diferentes escuelas (pai). Esto es 
una vergüenza, debido a todas estas razones; ellos han quebrado el cuerpo del guoshu. Para poder diseminar el guoshu en la sociedad, el guoshu debe ser estudiado usando métodos científicos, abiertos al público en general, investigando los diferentes sistemas y organizándolos de manera sistemática. Esta estrategia ha sido acordada por todos los artistas marciales.

(en Shi, 2008, 10: 11).

El párrafo anterior, dramático pero también optimista, muestra la seriedad de aquellos que se involucraron en este proyecto, así como la actitud abierta a nuevos métodos, en muchos casos foráneos, que permitirían alcanzar la meta propuesta por estos revolucionarios. Sin embargo, la afirmación de que todos los artistas marciales chinos se sumaron a este proyecto es exagerada, ya que solo las provincias bajo el control del Partido Nacionalista Chino y sus aliados se involucraron en el mismo (Morris, 2004).

Otras organizaciones privadas no asociadas a la Academia de Artes Nacionales también expresaron ideas similares, como lo evidencia la introducción de la revista Asociación Esencia Marcial Edición Especial (Jingwu Te Kan) publicada por la Asociación Atlética de la Esencia Marcial de Shanghái (la organización de artes marciales más influyente antes de la aparición de la Academia Central). En el primer volumen de la misma, escrito en 1923 se lee:

Los deportes occidentales permiten la discusión abierta y compartir experiencias. Sin embargo nuestra nación ha permanecido cerrada, envidiamos los avances ajenos y ocultamos nuestras desventajas. ¿A quién culpamos de que nuestros deportes estén declinando? N uestra Esencia Marcial se ha convertido en la razón para el avance de nuestro boxeo, combinando las escuelas del norte y del sur. No existe preferencia por ninguna. ¿Por qué no tratamos de exponer las características de cada escuela de manera abierta para entender y corregir sus desventajas? Y de esta manera mejorar nuestros deportes tradicionales de forma científica y sistematizada.

(en Shi, 2008, 4: 84).

\section{Forjando una nueva generación de artistas marciales}

Como se ha citado en la primera nota al pie, la práctica marcial en la antigüedad se asoció a los estudios de tipo académico. Es por ello que organizaciones asociadas a la Academia Central de Artes Nacionales, establecida en la ciudad de $N$ anjing en 1928 , incluyeron como parte del currículo materias como historia, biología o primeros auxilios entre otros (Chong, 1996). Unas de las razones la explica la publicación Artes Nacionales: Revista M ensual de la A cademia de Artes Nacionales de la Provincia de Zhejiang, al señalar que "Durante siglos aquellos expertos en guoshu, han descuidado la educación (wen); el analfabetismo no les ha permitido mejorar..." (en Shi, 2008, 10: 11). Tal vez el ejemplo más patente de este hecho se produjo en la Rebelión de los Boxeadores, los cuales fueron calificados como una turba de ignorantes supersticiosos. Al incluir métodos científicos, así como otras materias de estudio, los proponentes de las nuevas artes marciales buscaron disociarse de aquellos considerados como ignorantes (Morris, 2004).

La Asociación Atlética de la Esencia Marcial de Shanghái también incluyó diferentes asignaturas y departamentos adicionales a las artes marciales. Como 
ejemplo tenemos las secciones de estudios académicos y entretenimiento. Parte del departamento de estudios académicos lo conformaban las secciones editorial de deportes, literatura asiática y occidental, fotografía, estudios secretariales, caligrafía china, estudios médicos, pintura, etc. La asociación hizo uso extenso de la fotografía y de las ilustraciones en sus publicaciones, lo que ayudaría a la difusión de sus ideas y programas.

El departamento de entretenimiento constaba de balompié, lanzamiento de disco, billar, tirachinas, baloncesto, columpio (un deporte tradicional similar al que juegan los niños de Occidente), patinaje, dardos, música, etc. Otras sucursales de la asociación también tenían departamentos similares. Así, la asociación Esencia Marcial de Foshan estaba formada por nueve departamentos como por ejemplo gimnasia nacional, literatura, entretenimiento, editorial, comunicación pública, contabilidad, medicina, etc. (en Shi, 2008). Una de las aportaciones más importantes de esta asociación fue la inclusión sin restricciones de mujeres en sus programas, eliminando de plano costumbres que limitaban la participación de las mismas en actividades físicas, costumbre que aún se mantenía durante la dinastía Qing (Hui, 1919; Kennedy \& Guo, 2010).

O tro elemento considerado como importante en varios artículos de la época fue el de virtud marcial (wude). Este concepto fue promovido por la Asociación Esencia Marcial, por el periódico la Voz del Arte Nacional (Guoshu Sheng), publicado en 1928, así como por otras organizaciones que lo consideraban como algo muy importante en la formación de los artistas marciales. En la revista Espíritu Heroico (Xia Hun), aunque no se menciona el término wude de manera explícita, la descripción ofrece una idea de las expectativas que se tenían de los nuevos artistas marciales utilizando el concepto de "espíritu heroico":

¿Dónde están los que promueven Xia Hun? Un dicho antiguo sobre los que se unen a Xia dice que esto no es bueno para la ley o la sociedad, en realidad esto no es cierto. Xia ha ayudado a asegurar el poder político. ¿Q uién atrapará a las alimañas de la sociedad? Solo los Xia podrán hacerlo. ¿Q uién ayudará a los huérfanos y a las viudas? Solo los Xia. El comportamiento heroico de los Xia es como el Sol y la Luna en el cielo, los ríos en la tierra. ¿Quién podrá asumir el deber de un Xia? Solo los artistas marciales.

(en Shi, 2008, 28: 223)
EQUIPO DE BALONCESTO Y ORQUESTA DE LA ASOCIACIÓN ESENCIA MarCIAL, DE LA EDICIÓN ESPECIAL LIBRO DE ANIVERSARIO DE LA ESENCIA MARCIAL, 1919.
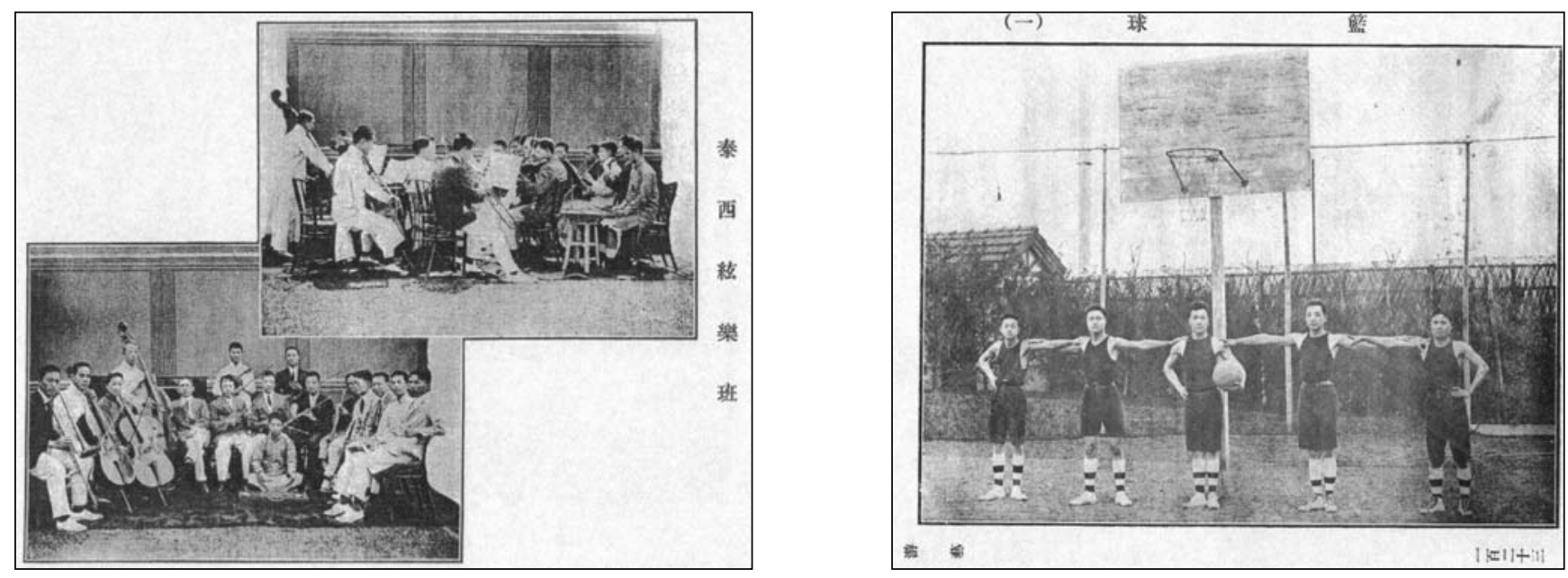
FOTOGRAFÍA SUPERIOR:

Práctica femenina de

RUTINAS A MANO VACÍA CON PAREJA EN LA ASOCIACIÓN

Esencia MarCIAL. (D)

HuANG W AN XIANG (I)

Cal Zhi Dan. Fotografía

INFERIOR: PrÁctICA

FEMEN INA DE RUTINAS

CON ARMAS EN PAREJA EN

LA ASOCIACIÓN ESEnCIA

MarCIaL (D) JIAN W EI

Qing (I) Chen ShI ChaO, UNA DE LAS PATROCINADORAS

MÁS IMPORTANTES DEL PROGRAMA PARA MUJERES DE

LA ASOCIACIÓN (KenNedY

\& GUO, 2010). EDICIÓN

ESPECIAL LIBRO DE

A NiverSARIO DE LA EsencIA MARCIAL, 1919.

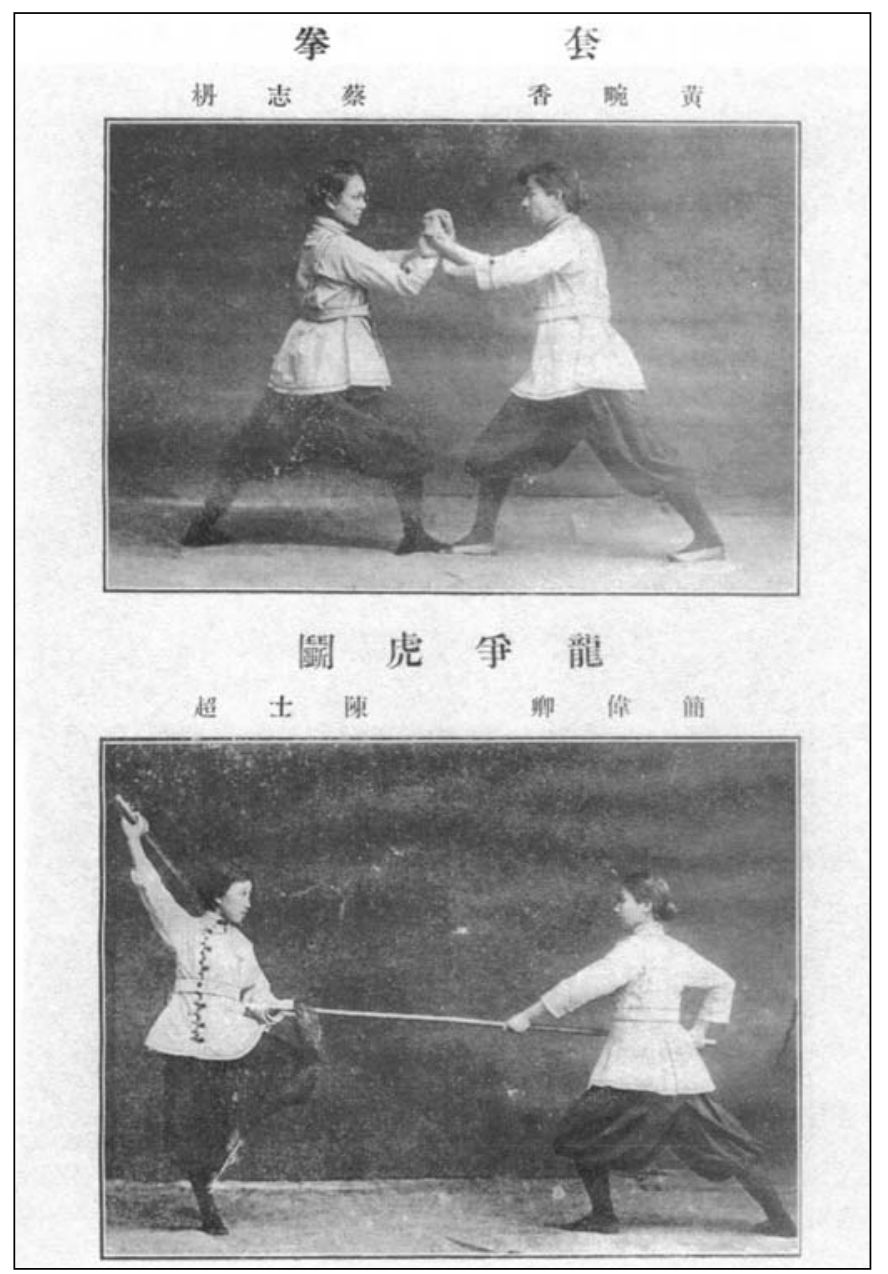
nipón.
El párrafo anterior muestra un rotundo cambio ideológico hacia los artistas marciales que contrasta con otras percepciones como la señalada en relación a la Rebelión de los Boxeadores. De vagabundos e ignorantes pasó a considerárseles héroes, con el sagrado deber de salvar a la nación. Este cambio fue promovido seguramente por el peligro que suponía Japón y su expansionismo agresivo en Asia, para el que los chinos estaban preparándose. Una situación que demuestra la razón fundamental para la práctica marcial, esto es, la preparación para la guerra, ya que China no contaba con los mismos recursos bélicos de su antagonista

\section{De artes de guerra a prácticas de competición}

El objetivo primordial de las artes marciales fue la habilidad en combate, con o sin armas. Este objetivo ha cambiado a lo largo de la historia debido al contexto cultural, social, político o económico en el que las artes de lucha se han desarrollado. Podemos citar como ejemplo las prohibiciones que no permitieron su práctica durante la dinasta Yuan (1271-1368), forzando a que estas solo se pudieran practicar en público en obras teatrales -situación que con seguridad influyó en su declive, como señala W ile (1999)-. Estas prácticas de exhibición fueron criticadas en la posterior dinastía Ming (1368-1644) por el general Qi Jiguang (Qi, 1560, ed. 1980). Del mismo modo, en la dinastía Qing las milicias que se unieron a la Rebelión de los Boxeadores, en muchos casos incluyeron elementos teatrales y mágicos en su práctica con nefastos resultados (Cohen, 1997). Tras la caída de los manchúes y el advenimiento de la república se buscó revitalizar la practica marcial, tomando como base los antiguos exámenes militares imperiales que se habían celebrado entre las dinastías Tang (618-907) y Qing (1644-1912) (Acevedo, Gutiérrez, $\&$ Cheung, 2010).

En la Revista de la Academia Central de Artes Nacionales (Zhongyang Guoshu Guan Hui Kan) se explican los motivos por los que la competición abierta ayudaría a revitalizar las artes marciales chinas:

Nuestra nación ha existido durante más de 5000 años, desafortunadamente nuestra cultura está disminuyendo y nuestra economía está en muy mala situación [...] nuestra nación ha descuidado el entrenamiento físico debido a la falta de sistemas de competición. Esta ha sido la razón por la que estas prácticas se han debilitado. El impacto de esta situación ha causado de manera indirecta que nuestra gente no haya acudido a la defensa de nuestra nación.

(en Shi, 2008, 8: 383) 
La revista también describe en términos generales cómo se organizaron los exámenes de artes nacionales. Los exámenes estaban divididos en tres niveles, a saber: nacional, provincial y local. Cada nivel era organizado por la academia, con el gobierno nacional estableciendo la fecha para su realización. En el examen provincial, el gobierno nacional le informaría al gobierno provincial de la fecha para este examen, quien procedería igualmente con el gobierno a nivel local. Los candidatos deberían ser aprobados por dos calificadores en el examen local. Una vez aprobado, el candidato recibiría un certificado de recomendación que podría usar para presentarse al examen provincial; de la misma manera, el candidato debería pasar por un proceso similar en el examen provincial y así ganar el derecho a presentarse al examen nacional.

La academia tenía diferentes sucursales en las diferentes provincias pero no en cada localidad, por esta razón aquellos estudiantes de la Academia de Artes Nacionales que desearan presentarse al examen local pero no podían regresar a su lugar de origen se les permitiría presentarse al examen organizado por la sucursal de la academia más cercana. Los gastos de viaje de los candidatos eran cubiertos por el gobierno provincial o local de origen del candidato. Los títulos de los ganadores de los exámenes eran: a nivel local y de ciudad, héroes (xian); a nivel de pueblos el primer puesto otorgaba un certificado de reconocimiento. El número de candidatos que podrían recibir este título era de tres a diez, en el caso de una ciudad solo de dos a cinco candidatos podían recibirlo. A nivel provincial y de distrito especial, se les otorgaba el titulo de guerrero (wu shi), recibiendo un certificado de reconocimiento. El número de candidatos que recibirían este título era de diez a cuarenta, y en el caso de distritos especiales solo de cinco a quince. En el examen nacional, se dividió a los finalistas en las siguientes clasificaciones: primera clase (jia deng) subdividida en orden descendente con los títulos defensor (han wei), seguidor (fu wei), asistente (yi wei). La segunda clase (yi deng) permitía de veinte a treinta finalistas con el titulo de oficial junior (xiao wei). Por último, la tercera clase (bing deng) estaba conformada entre ochenta y dos y ciento veinte finalistas con el titulo de valiente (yong shi).

Antes del examen nacional cada provincia y distrito especial debía informar del número de candidatos con un periodo anticipado mínimo de dos meses. Con la misma antelación, para el examen provincial, las ciudades y pueblos debían informar del número de candidatos a la sucursal provincial. Para el examen a nivel local el plazo disminuía a un mes. La fecha del examen nacional era el 1 de octubre de cada año, los exámenes provinciales se realizaban el 1 de abril y a nivel local se celebraban el 1 de diciembre.
FOTOGRAFÍA SU PERIOR:

PRÁctica de armas EN PAREJA, BASTÓN (GUN) FRENTE A ESPADAS GANCHO (HU SHOU GOU). FOTOGRAFÍA IN FERIOR: PRACTICA DE LA FORMA DE LOS CINCO ELEMENTOS DE BOXEO DE LA MENTE E INTENCIÓN (XINGYI Wu XING). Revista Anual de Artes nacionales de la Provincia de JIANGSU, JULIO 1929.

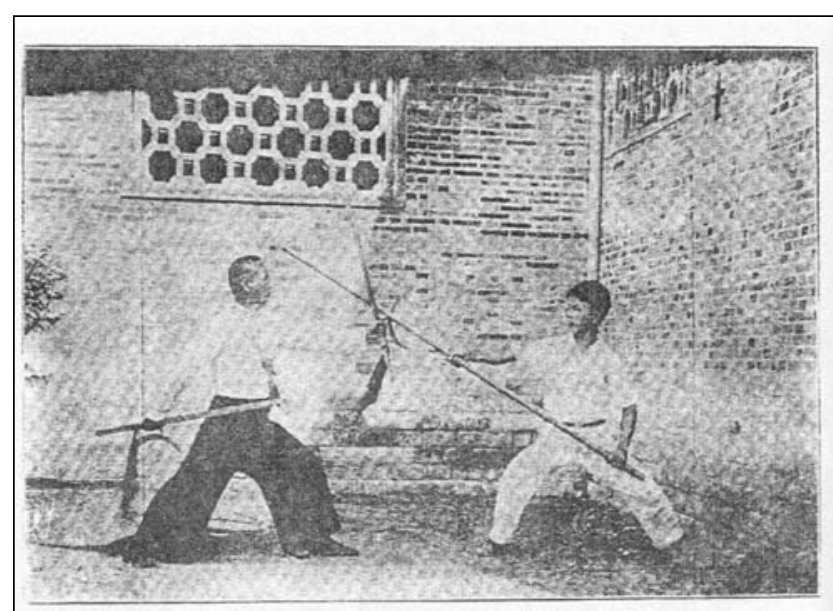

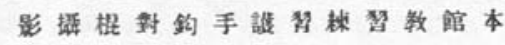

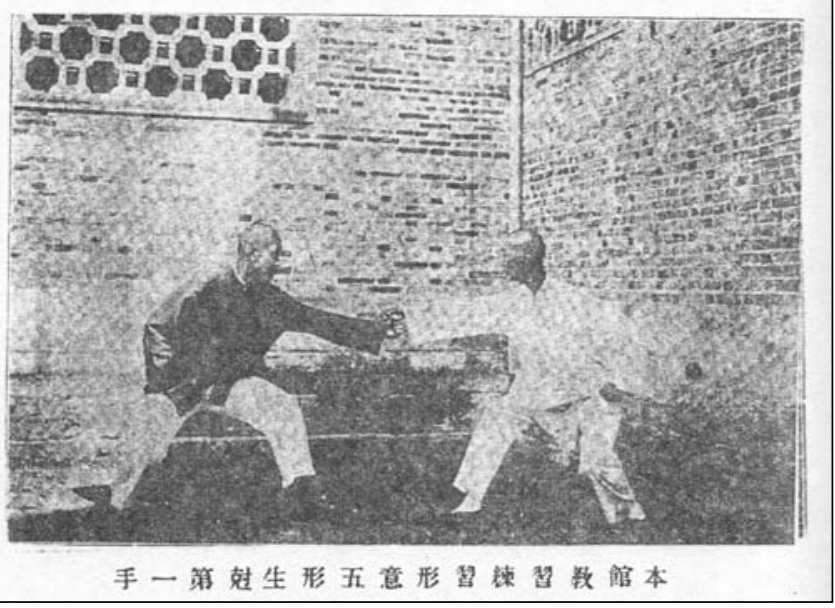


"LOS HOMBRES DE ACERO NO PUEDEN SER DERROTADOS". EDICIÓN ESPECIAL LIBRO DE Aniversario de LA EsenCia MARCIAL, 1919.
Los temas del examen nacional incluían en primer lugar guoshu, conformado por combates de boxeo chino (quan jiao ke), lucha (shuai jiao ke) y estudio de armas (qi xie ke). Este último evento incluía lanza, bastón, sable y espada de dos filos. El candidato debía superar las pruebas de boxeo, lucha y una de las pruebas de combate con armas. En el examen nacional era obligatorio practicar boxeo y lucha, y los candidatos podían escoger una de las cuatro armas. El candidato era evaluado no solo por sus conocimientos en los estudios nacionales sino también por su ética. En segundo lugar, el examen nacional incluía estudios nacionales (guo xue) dentro de los cuales estaban, los Tres Principios de la Gente (San min zhu yi), historia de las artes nacionales y bases de fisiología e higiene. En los exámenes locales y provinciales el componente más importante era el combate a mano desnuda contra un oponente (dui shi quan jiao). El candidato debía pasar, además del boxeo, una más de las restantes pruebas.

Los exámenes tenían dos partes, un examen preliminar (yu shi) y el examen formal (zheng shi); fallar el examen preliminar descalificaba al candidato. El examen preliminar nacional consistía en demostrar habilidad en boxeo, lucha y armas. En los exámenes provinciales y locales se seguía el mismo proceso. Durante el examen formal se utilizaba un sistema de sorteo para seleccionar los contrincantes en las categorías descritas, a cada candidato se le asignaba un número y los ganadores de sus respectivos combates eran una vez más emparejados con otro oponente siguiendo el mismo sistema.

Las pruebas de combate a mano vacía se llevaban a cabo en tres asaltos, resultando vencedor el ganador de dos de los mismos. En el evento de lucha la persona que terminaba en el suelo otorgaba puntos a su oponente, si los dos luchadores caían al mismo tiempo se consideraba un empate, si alguno de los adversarios no podía terminar el asalto perdía el combate, y evitar pelear era castigado con la eliminación. En la lucha con armas un ataque a las manos o brazos otorgaba puntos, y si los oponentes se golpeaban al mismo tiempo se consideraba un empate. Se castigaban las técnicas ilegales 0 aquellas dirigidas a zonas consideradas como prohibidas.

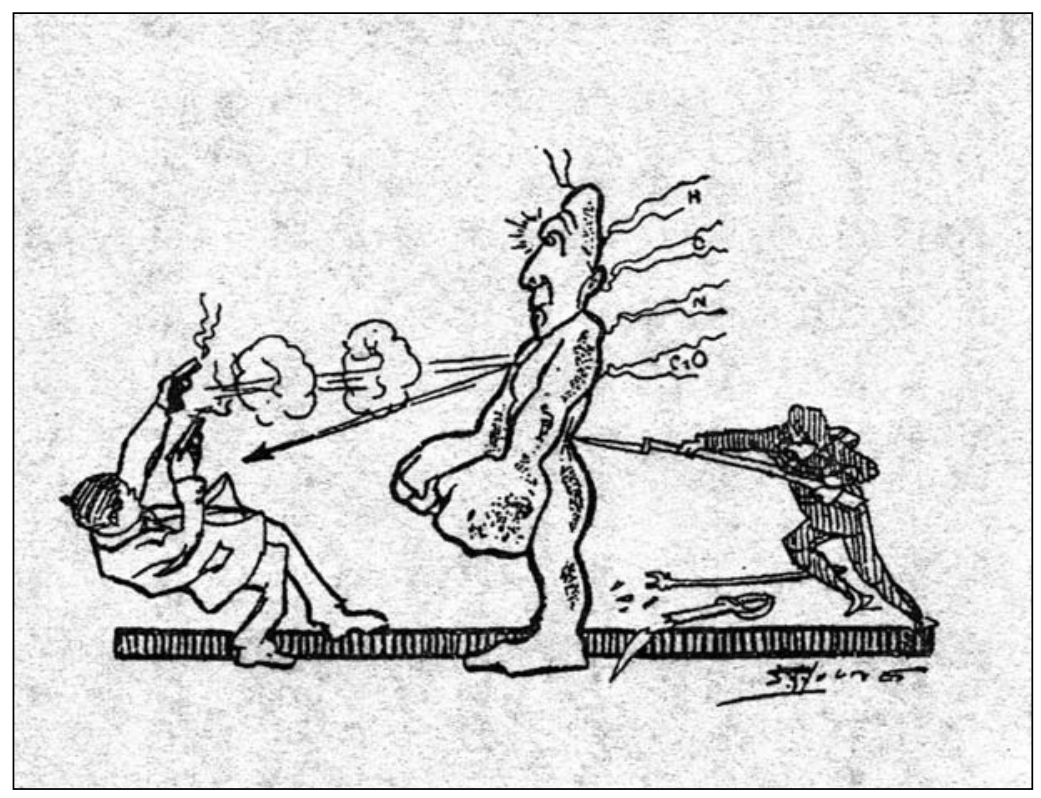

Los eventos de combate a mano vacía requerían el uso de protecciones. Estas no se especifican en el texto original, aunque en fotos de la época los combatientes vestían un protector de pecho similar a los usados en el béisbol de la época. Las reglas, tal y como aparecen en el texto original, son muy generales y en ocasiones ambiguas. Además de lo descrito, en los combates con armas también se portaba un casco improvisado con lo que parece madera o cuero y malla para proteger el rostro. En el texto original tampoco se explican claramente las reglas, las cuales fueron modificadas en el segundo examen nacional. 


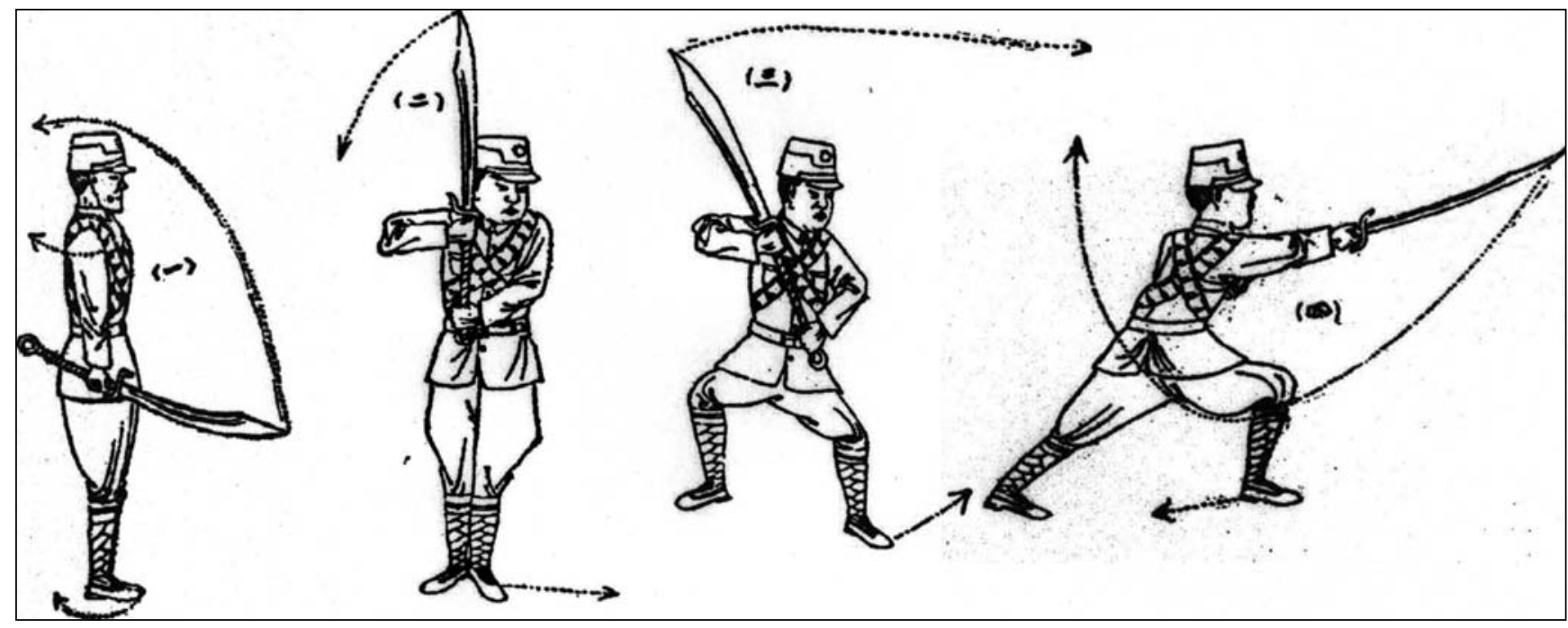

\section{China prepara su ejército}

Durante el periodo de la historia japonesa conocido como Restauración Meiji (1868-1912), las fuerzas armadas niponas fueron modernizadas siguiendo modelos occidentales, aunque también se preservaron tradiciones marciales autóctonas. Los japoneses asimilaron y mejoraron lo aprendido de naciones como Francia y Alemania (Norman, 1905); como ejemplo de este hecho podemos citar la guerra Ruso-japonesa (1904-1905), donde se produjo la segunda victoria japonesa en una década sobre un gran país del continente euroasiático. Un arma a la que los observadores occidentales señalaron como decisiva, además de la artillería, fue el espíritu generado a través del entrenamiento en el uso de la bayoneta, que los japoneses utilizaban con mortal efectividad. Las lecciones aprendidas de este conflicto fueron estudiadas por los ejércitos de Inglaterra y Francia (Engen, 2006).

Es durante este periodo de modernización cuando se inaugura la Escuela Militar Toyama (Rikugun Toyama Gakko), en la que se desarrollaron métodos de combate con sable y bayoneta que se enseñarían a los soldados, además del entrenamiento en el "arte suave" (ju jutsu) y la lucha japonesa (sumo) (Cook, 2004) ${ }^{6}$. Un dato interesante ocurrió en 1904 en la ciudad de Shanghái, cuando un grupo británico de esgrima con bayoneta participó en un concurso amistoso frente a su contraparte japonés. A pesar de que los británicos eran las fuerzas occidentales más adeptas al uso de esta arma, fueron derrotados por los japoneses (Svinth, 1999).

Paralelamente China también había comenzado a modernizarse a finales de la dinastía Qing, en un periodo conocido como Movimiento de Auto Fortalecimiento (1861-1895), aunque no con el mismo éxito que Japón. En 1901 el gobierno manchú comenzó a enviar estudiantes a la ciudad de Tokio para que estudiaran las tácticas policiales niponas. Tras la caída de la dinastía Qing y el ascenso al

6 Un hecho importante que merece la pena entender es que aun hoy en día, y sin importar los avances tecnológicos militares, el entrenamiento en técnicas de lucha con y sin armas es considerado como algo importante en la preparación del soldado, generando una actitud de "hacer 0 morir" en el campo de batalla (Svinth \& Sucher, 2000).
RUTINA CON DA DAO

INCLUIDA EN EL LIBRO

TÉCNICAS DE BAYONETA

y Sable Chin os. Revista

Semanal de ARtes

NACIONALES, 1935. 


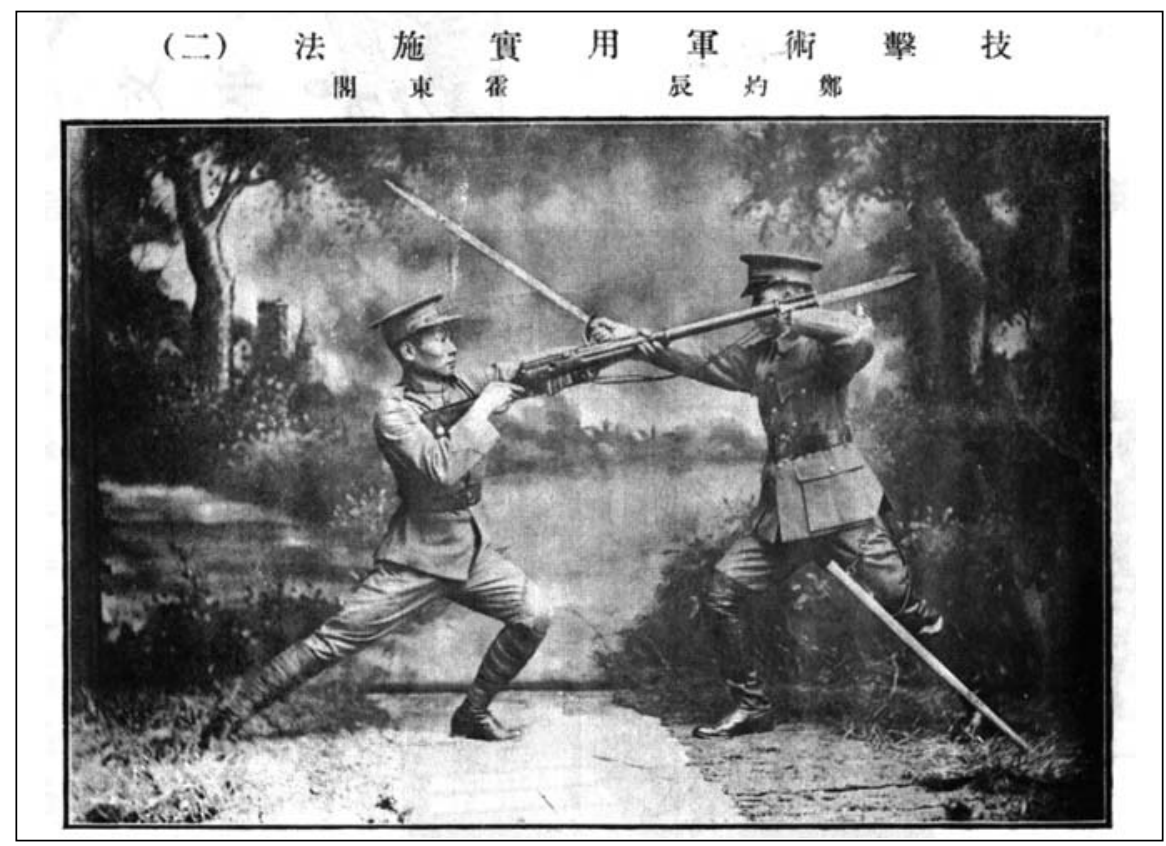

poder del gobierno nacionalista, se buscó aprender de los modelos policiales austriacos y estadounidenses (W akeman, 1992). En 1909, un grupo de estudiantes chinos establecidos en la ciudad de Tokio, miembros de la Liga China Unida7, editaron la revista Estudios Marciales (Wu Xue). Los objetivos de la misma fueron "Promover el espíritu marcial, el estudio de las artes marciales, la discusión de métodos para el entrenamiento de nuestros soldados, reforzar la educación militar, discutir acerca de armamento y la marina [...]" (en Shi, 2008, 29: 227).

EJEMPLO DE HABILIDADES DE ATAQUE APLICADAS EN EL E)ÉRCITO, PRACTICADAS EN

la Asociación Esencia Marcial. (D) Zheng Zhuo Chen (I) Huo Dong Ge. EDICIÓN ESPECIAL LIBRO DE AniverSARIO de LA ESEnCIA Marcial, 1919.

Con la apertura de la Academia Militar para Oficiales Chinos del Partido Nacionalista (Zhongguo Guomindang Lujun Junguan Xuexiao), establecida en 1924, las fuerzas armadas chinas también empezaron a modernizarse. Se publicaron diferentes artículos enseñando los métodos de combate con bayoneta y sable a la población civil y al ejército. Es importante mencionar que los chinos estudiaron los métodos de lucha japoneses para así entender sus debilidades y contrarrestarlos con los propios, siguiendo el ejemplo del general de la dinastía Ming, Qi Jiguang, quien hizo lo mismo frente a los piratas japoneses que asolaban las costas de China (A cevedo, Gutiérrez, \& Cheung, 2010). De hecho, los esfuerzos de Qi fueron el tema de un artículo publicado en 1934 en la Revista Semanal de Artes Nacionales (Guoshu Zhou Kan). En un artículo titulado "Discutiendo las Artes $N$ acionales", publicado en el mismo año y revista, se discute el propósito militar del guoshu:

Debe ser practicado por el ejército, es una forma de ataque y defensa para derrotar a los enemigos de la patria. Debe ser promovido con la misma importancia que el amor a la patria y el espíritu marcial. Últimamente el guoshu se ha usado contra el bushido. El guoshu debe ser practicado con atención y su aplicación está en el campo de batalla. El practicante fortalecerá su cuerpo para transformarse en un hombre de acero, además de su coraje. Guoshu requiere de práctica constante para dominarlo y poder utilizarlo de manera eficiente; algunos practicantes han ignorado u omitido la aplicación de sus rutinas, lo cual es una lástima [...]. Para fortalecer la nación se debe tener una raza fuerte, para tener una raza fuerte se debe tener un cuerpo fuerte, y para tener un cuerpo fuerte se debe practicar guoshu; aquellos que practican guoshu no lo hacen para defenderse sino para defender a la nación. Todos aquellos que se han convertido en los hombres de hierro de hoy, se han preparado para ser las armas del mañana.

(en Shi, 2008, 12: 59)

7 Establecida por el revolucionario chino Sun Yat-sen (Sun Yixian, 1866 -1925). 
En la misma revista se comenzó una serie de artículos dedicados a las técnicas de cortar y apuñalar. En el primero de los mismos, escrito por el editor de la revista jin Jing Zhong y titulado "Estilo de bayoneta china"8, se señala que en los XVIII juegos deportivos, para promover el espíritu marcial en la población, se incluyeron demostraciones de bastón realizadas por boyscouts, ejercicios de taijiquan realizados por estudiantes de escuela primaria, y exhibiciones de sable cortante y de bayoneta china por equipos de seguridad. El equipo de seguridad (bao an dui) entrenó durante un año, siendo invitado al evento para promover el nacionalismo, la transformación de la población civil en "hombres de acero" y el entrenamiento en las armas del país; de este modo se lograría vengar el honor de la nación.

Finalmente, en la misma Revista Semanal de Artes Nacionales, Li Q in Min publicó un artículo en 1934 con una breve introducción acerca de la importancia y razones por las cuales el uso del sable a dos manos era una habilidad importante en la preparación de los soldados chinos, como se puede leer a continuación:

Las unidades de sables/cuchillos (da dao dui) largos lucharon contra los japoneses en Shanghái. Durante este evento, especialistas en su uso fueron parte de las tropas chinas. Estos hombres lucharon a corta distancia, debido a la falta de armamento moderno, los soldados de esta unidad fueron seleccionados cuidadosamente. Las tropas armadas con sables largos estaban divididas en diferentes unidades como eran los Especialistas en Sables Largos considerados como la unidad elite, las Unidades Combinadas de Sables Largos, las cuales además de estar armadas con el sable también tenían otras armas como rifles. Estas unidades usaron los rifles cuando se encontraban a distancia, y una vez estando cerca harían uso de la bayoneta o del sable. Finalmente, el Grupo Armado de Sables Largos tenía en su arsenal pistolas de mano, granadas, dagas en la pierna y el sable. Durante los enfrentamientos a corta distancia el uso del sable a dos manos era un arma ideal, en caso de perderlo el soldado podría usar su cuchillo u otro tipo de arma para luchar. Las Unidades de Sables Largos sirvieron en el campo de batalla del s. XX, debido a la necesidad del momento, estas armas se entrenaron de manera práctica, requiriendo de mucho coraje para usarlas. Aplicando las artes nacionales en el campo de batalla para poder alcanzar la victoria, utilizando la habilidad en el manejo del sable por los miembros del ejército del noroeste, lo cual se ha convertido en un elemento esencial de nuestras Artes Nacionales.

(en Shi, 2008, 24: 30).

\section{Conclusiones}

El presente artículo ha mostrado diferentes fuentes primarias para el estudio de las artes marciales chinas durante el periodo republicano. En ellas se aprecia el

8 Los orígenes del estilo de bayoneta china se sitúan en 1925. En aquel momento las habilidades del ejército no eran lo suficientemente buenas, y debido a la falta de práctica los soldados no tenían coraje para luchar. Por tanto, se comenzaron a estudiar e investigar las habilidades de combate con bayoneta. Tras un periodo de cuatro años de estudios, Jin Jing Zhong publicó un libro de técnicas de sable y bayoneta titulado Técnicas de Bayoneta y Sable Chinos (Zhonghua Ci Qiang Shu Yu Da Dao Shu). 
fervor nacionalista y el deseo de proteger la nación a través de la práctica marcial. También se demuestra que el concepto de guoshu era más amplio que el concepto moderno de wushu.

Las informaciones contenidas en estas publicaciones tienen un valor innegable. Entre las mismas hemos destacado cómo la amenaza del expansionismo nipón forzó la asimilación de métodos organizativos occidentales y el estudio de las técnicas de lucha japoneses para encontrar sus debilidades. Asimismo el llamamiento a la población civil para que se fortaleciera y defendiese a la nación a través de la práctica del guoshu, para lo cual, entre otras iniciativas, se instauraron los exámenes de guoshu. Por último, también se ha destacado que la promoción del uso del rifle-bayoneta y el sable a dos manos fue una necesidad apremiante debido a la falta de armamento más moderno para las tropas chinas, las cuales debieron hacer uso de su ingenio para afrontar la amenaza nipona.

Además de las señaladas, los treinta volúmenes de la Colección de Periódicos de Artes Nacionales del Periodo Republicano guardan muchas otras informaciones relevantes para los estudiosos de las artes marciales chinas, que con toda probabilidad seguirán saliendo a la luz durante los próximos años.

\section{REFEREN CIAS}

Acevedo, W., Gutiérrez, C., \& Cheung, M. (2010). Breve Historia del Kung Fu. Madrid: Ediciones Nowtilus.

An, T. (s.f.). W ushu Needs Name Rectification. Kung Fu Magazine.com. Disponible en http://ezine.kungfumagazine.com/ezine/article.php?article=581. [Consulta 22/12/2010].

Brownell, S. (1995). Training the Body for China. Chicago: The University of Chicago Press.

Chong, Y. (1996). Zhongyang Guoshu Guan Shi [Historia de la A cademia Central de Artes Nacionales]. Hefei, Anhui: Huangshan Press.

Cohen, P. A. (1997). History in Three Keys. New York: Columbia University Press.

Cook, H. (2004). Some Thoughts on the Evolution of Traditional Karate. Classical Fighting Arts, 2, 14-15.

Couch, J. (Noviembre de 2009). Chinese Martial Arts in 19th century China. Recuperado el 23 de 0 ctubre de 2010, de Martial History: http://martialhistory.com/2009/11/chinese-martial-arts-in-19th-century-china/

Engen, R. (2006). Steel A gainst Fire: The Bayonet in the First W orld W ar. Journal of Military and Strategic Studies, 8(3), 8-10.

Hui, J. T. (1919, reed. 2008). Jingwu Benji [A niversario de la Esencia Marcial]. Taiwan: Lion Books.

Kennedy, B., \& Guo, E. (2010). Jingwu The School that Transformed Kung Fu. California: Blue Snake Books.

Ma, M. (2009). Reconstructing China's Indigenous Physical Culture. Journal of Chinese Martial Studies, 1, 8-31.

Zedong, M. (Abril de 1917). A Study of Physical Education. En Selected Works of Mao Tse-tung. Disponible en http://www.cddc.vt.edu/marxists/refe- 
rence/archive/mao/selected-works/volume-6/mswv6_01.htm. [Consulta 25/10/2010].

Morris, A. D. (2004). Marrow of the Nation. California: University of California Press.

Norman, F. J. (1905, ed. 2006). The Fighting Man of Japan. London: Dover Publications.

Qi, J. (1560) Ji Xiao Xin Shu [Nuevo Libro de Disciplina Efectiva]. Edición de Ma, M. (1980). Beijing: Renmin Tiyu Chubanshe.

Shi, Y. X. (2008). Ming Guo Guoshu Qi Kan Wen Xian Ji Cheng (Colección de Periódicos de Artes Nacionales del Periodo Republicano). Beijing: Zhongguo Shudian.

Svinth, J. R. (1999). An Introduction to Training in Rifle-Bayonet Fighting in the US Military. Journal of Non-lethal Combatives. Disponible en http:// ejmas.com/jnc/jncart_svinth3_1199.htm. [Consulta 29/10/2010].

Svinth, J. \& Sucher, J. (escritores); Fischier, S., Kondo, T., \& Sucher, J. (Dirección). (2000). Martial Arts: The Real Story [DVD]. Hastings on Hudson, NY: Pacific Street Films.

W akeman, F. (1992). Police Advicers and the Nacionalist Chinese Secret Service. Modern China, 18(2), 107-137.

W ile, D. (1999). T'ai Ch'is Ancestors The Making on an Internal Martial Art. New York: Sweet Ch'i Press.

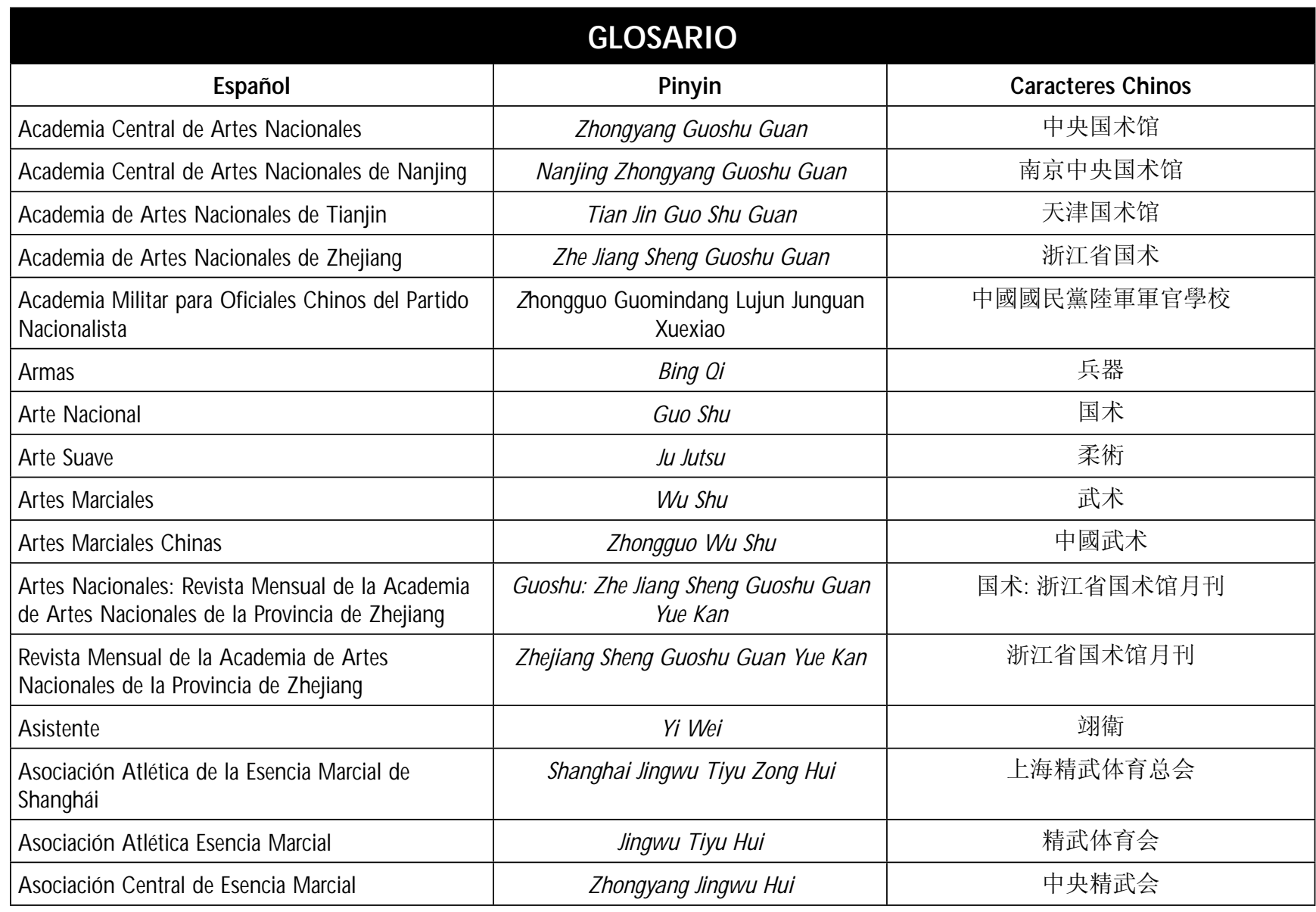




\section{GLOSARIO}

Asociación Central de la Esencia Marcial de Shanghái

Asociación China de Artes Marciales de Shanghái

Asociación China de Artes Marciales de Shanghái,

3a Reunión de Miembros Edición Especial

Asociación China de Deportes Nacionales

Asociación Esencia Marcial Edición Especial

Asociación para el Estudios de Deportes Nacionales

Asociación para la Promoción de las Artes

Nacionales de la Provincia de Shanxi

Bastón

Biblioteca de la Universidad de Beijing

Biblioteca de la Universidad de Xiamen

Biblioteca de Shanghái

Biblioteca Nacional

Boxeo Chino

Bushido

Casa Editorial de Nanjing

Central

Centro de Entrenamiento de Artes Nacionales de Hunan

Centro de Investigación de Artes Nacionales

Condado de Cheng Provincia de Shanxi

Centro de Investigación Deportiva de Beijing

Ciudad

Colección de Discursos de Artes Nacionales del

Señor Zhang Zhi Jiang

Colección de Periódicos de Artes Nacionales del Periodo Republicano

Combate a Mano Desnuda contra un Oponente

Cortar y Apuñalar

Defensor

Departamento Académico Deportivo de la Esencia

Marcial de Xiamen

Departamento de Deportes de la Universidad de Zhejiang

Deportes

Deportes Nacionales

Discutiendo las Artes Nacionales

Edición Especial de Artes Nacionales del Condado de Yin

Edición Especial de la Conferencia Recreativa de Artes Nacionales de Zhejiang
Shang Hai Zhong Yang Jing W u Hui

Shanghai Zhong Hua Wu Shu Hui

Shanghai Zhong Hua W u Shu Hui Di San Jie Zheng Qiu Da Hui Te Kan

Zhong Hua Quan Guo Ti Yu Xie Jing Hui Jingwu Te Kan Guo ji Yan Jiu Hui

Shanxi Sheng Guo Shu Cu Jing Hui

Gun

棍

北京大学图书馆

Beijing Daxue Tushu Guan

Xiamen Daxue Tushu Guan

Shanghai Tuxhu Guan

Guojia Tushu Guan

Quan Shu

Wu Shi Dao

Nan Jing Chu Ban She

Zhongyang

Hu Nan Guo Shu Xun Lian Suo

夏门大学图书馆

上海图书馆

国家图书馆

拳术

武士道

南京出版社

中央

湖南国术训练所

Shan Xi Sheng Cheng Gu Xian Guo Shu Yan Jiu Hui

Ti Yu Yan Jiu She Bian Ji

Shi

Zhang Zhi Jiang Xian Sheng Guo Shu Yan Lun Ji

Mingguo Guoshu Qi Kan Wenxian

Xia Men Jing W u Ti Yu Hui Xue Shu Bu

Zhejiang Daxue Tiyu She

Yin Xian Xian Guo Shu Guan Yi Zhou

Zhe Jiang Guo Shu You Yi Da Hui Hui Kan jicheng

Dui Shi Quan Jiao

$\mathrm{Pi} \mathrm{Ci}$

Han Wei

浙江大学体育社

体育

国粹体育

國術漫談 Nian Ji Nian Kan

民国国术期刊文献集成

對試拳腳

䢃束

捍衛

夏门精武体育會学术部

Guo Cui Tiyu

Guoshu Man Tan

鄞县县国术馆一周年纪念刊

浙江国术游艺大会汇刊 


\section{GLOSARIO}

Edición Especial del Movimiento de Artes $\mathrm{N}$ acionales

Edición Especial del Primer Examen Nacional

Edición Especial del Segundo Examen Nacional de Artes Nacionales

Educación

Entretenimiento

Equipo de Seguridad

Escuela de artes Nacionales de Beijing

Escuela de Artes Nacionales de la Municipalidad de Shanghái

Escuela de Artes Nacionales de la Provincia de

Zhejiang

Escuela de Deportes de Li en Hong Kong

Escuela de Esencia Nacional

Escuelas

Esencia Marcial

Esencia Marcial de Foshan

Esencia Marcial de Xiamen

Esencia Marcial Pictórica

Espada

Especialista en Sables/Cuchillos Largos

Espíritu Heroico

Estadio Deportivo Jiang de Shanghái

Estilo de Bayoneta China

Estudio de Armas

Estudio de Bastón Largo

Estudio de Boxeo Chino

Estudio de Espada de Doble Filo

Estudio de Lucha China

Estudios Académicos

Estudios Marciales

Estudios Nacionales

Examen Formal

Examen Preliminar

Exámenes de Artes Nacionales

Fisiología

Gimnasia Nacional

Grupo Armado de Cuchillos Largos

Guerrero

Habilidad en el uso del Sable/Cuchillo Largo
Guo Shu - Dong De Te Kan

Di Yi Ci Guo Kao Te Kan

Zhong Yang Di Er Jie Guo Shu Guo Kao Zhuan Kan

Wen

You Yi Bu

Bao An Dui

Beijing Te Bie Shi Guo Shu Guan

Shang Hai Shi Guo Shu Guan

Zhe Jiang Sheng Guo Shu Guan

Xiang Gang Li Shi Jian Shen Xue Yuan

Guo Cui

Pai

Jing $W u$

Foshan Jingwu

Jing Wu (Xia Men)

Jingwu Hua Bao

jian

Zhuan Yong Da Dao

Xia Hun

Shang Hai Jiang Wan Ti Yu Chang

Zhonghua Ci Qiang Shu

Qi Xie Ke

Gun Shu Ke

Quan Jiao Ke

Jian Shu Ke

Shuai Jiao Ke

Wen Shu Bu

Wu Xue

Guo Xue

Zheng Shi

Yu Shi

Guo Shu Kao Shi

Sheng Li Da Yao

Guo Cao

Quan Shen Wu Qi Da Dao Dui

Wu Shi

Dao Shu
国术-动的特刊

第一次国考特刊

中央第二届国术国考专刊

文

游艺部

保安隊

北平特别市国术馆

上海市国术馆

浙江省国术馆

香港李氏健身学院

國粹

派

精武

佛山精武

精武夏门

精武画报

劍

專用大刀

侠魂

上海江湾体育场

中華刺槍術

器械科

棍术科

拳腳科

劍术科

率角科

文书部

武学

國學

正試

預試

國術考試

生理大要

国操

全身武器大刀隊

武士

刀術 


\section{GLOSARIO}

\begin{tabular}{|c|c|c|}
\hline Habilidades de Ataque & Ji Ji & 技击 \\
\hline Habilidades Militares & WuYi & 武㙯 \\
\hline Héroes & Xian & 縣 \\
\hline Higiene & Wei Sheng Yao Yi & 衛生要義 \\
\hline Historia de las Artes Nacionales & Guo Shu Shi & 國術史 \\
\hline Hombre de Acero & Tie Han & 鐵漢 \\
\hline Hombres Enfermos de Asia del Este & Dong Ya Bing Fu & 东亚病夫 \\
\hline Juego del Gallito & Jian Zi & 建子 \\
\hline Lanza & Qiang & 槍 \\
\hline Las U Itimas Reglas de Artes Nacionales & Zui Xing Guo Shu Gui Ze & 最新国术规则 \\
\hline Libro de Aniversario de la Esencia Marcial & Jing W u Ben Ji & 精武本纪 \\
\hline Liga China Unida & Zhong Guo Tong Meng Hui & 中國同盟會 \\
\hline Local & Xian Kao & 縣考 \\
\hline Los Anales de Confucio & Lun Yu & 論語 \\
\hline M ejoramiento de las Habilidades de Ataque & Ji Ji Gai Jing & 技击改进 \\
\hline Movimiento de Auto Fortalecimiento & Yang Wu Yun Dong & 洋務運動 \\
\hline Nacional & Guo Kao & 國考 \\
\hline Nuevas Artes Marciales de China & Zhong Hua Xin Wu Shu & 中華新武術 \\
\hline Nuevo Movimiento Cultural & Xin Wenhua Yundong & 新文化運動 \\
\hline Oficial Junior & Xiao Wei & 校衛 \\
\hline Organización de Artes Marciales Chinas & Zhonghua Wushu Hui & 中华武术会 \\
\hline Organización de Caballeros Marciales & Zhonghua Wuxia Hui & 中华武俠会 \\
\hline Partido Nacionalista Chino & Guo M in Dang & 國民黨 \\
\hline Periódico Mensual de la Esencia Marcial de Foshan & Foshan Jingwu Yue Kan & 佛山精武月刊 \\
\hline Periódicos/Revistas & Qi Kan & 期刊 \\
\hline Practica con O ponente & Dui Shou & 对手 \\
\hline Primavera y O toño de la Esencia Marcial & Jing W u Chun Qiu & 精武春秋 \\
\hline Primer Puesto & Zhuang Shi & 壯士 \\
\hline Primera Clase & Jia Deng & 甲等 \\
\hline Provincial & Sheng Kao & 省考 \\
\hline Publicación Mensual de Búsqueda de la Verdad & Xia Hun Qiu Shi Yue Kan & 侠魂求是月刊 \\
\hline Publicación Trimestral de la Búsqueda de la Verdad & Qiu Shi Ji Kan & 求是季刊 \\
\hline Raza & Zhong & 種 \\
\hline Registro de Artes Nacionales & Guo Shu Zhan Ji & 国术战迹 \\
\hline Reglas de Artes Nacionales & Guo Shu Gui Ze & 国术规则 \\
\hline Remedio & Hui Chun Dan & 回春丹 \\
\hline Reporte de la Asociación de Esencia Marcial & Jing Wu Cong Bao & 精武从报 \\
\hline Restauración M eiji & Ming Zhi Wei Xin & 明治維新 \\
\hline $\begin{array}{l}\text { Revista Anual de Artes } N \text { acionales de la Provincia } \\
\text { de Jiangsu }\end{array}$ & Jiang Su Sheng Guo Shu Guan Nian Kan & 江苏省国术馆年刊 \\
\hline
\end{tabular}




\section{GLOSARIO}

\begin{tabular}{|c|c|c|}
\hline Revista Anual de la Esencia Marcial & Jing Wu Nian Bao & 精武年报 \\
\hline Revista Central de Artes Nacionales & Zhong Yang Guo Shu Xun Kan & 中央国术旬刊 \\
\hline Revista de Deportes & Ti Yu Cong Kan & 体育丛刊 \\
\hline Revista de Deportes y Artes Nacionales de Shanxi & Shan Xi Guo Shu Ti Yu Xun Kan & 山西国术体育旬刊 \\
\hline Revista de la Academia Central de Artes Nacionales & Zhongyang Guoshu Guan Hui Kan & 中央国术馆汇刊 \\
\hline Revista de la Esencia Marcial & Jing Wu Za Zhi Xiang Gang & 精武杂志 (香港) \\
\hline Revista Esencia Marcial & Jingwu Za Zhi & 精武杂志 \\
\hline Revista Mensual de Artes Nacionales de Shanxi & Guo Shu Yue Kan (Shan Xi) & 国术月刊 (山西) \\
\hline Revista Mensual de Artes Nacionales Unidas & Guo Shu Tong Yi Yue Kan & 国术统一月刊 \\
\hline $\begin{array}{l}\text { Revista Quincenal de Artes Nacionales de la } \\
\text { provincia de Hunan }\end{array}$ & Guo Shu Ban Yue Kan Hu Nan & 国术半月刊湖南 \\
\hline Revista Quincenal de Artes Nacionales de Shanghái & Guo Shu Ban Yue Kan - Shang Hai & 国术半月刊-上海 \\
\hline Revista Semanal de Artes N acionales & Guo Shu Zhou Kan & 国术周刊 \\
\hline Sable & Dao & 刀 \\
\hline Sable Cortante & Pi Dao & 䢃刀 \\
\hline Salud y Belleza & Jian Yu M ei & 健与美 \\
\hline Seguidor & Fu Wei & 輔衛 \\
\hline Segunda Clase & Yi Deng & 乙等 \\
\hline $\begin{array}{l}\text { Sociedad de la Revista Mensual de Artes } N \text { acionales } \\
\text { Unidas de Shanghái }\end{array}$ & Shang Hai Guo Shu Tong Yi Yue Kan She & 上海国术统一月刊社 \\
\hline $\begin{array}{l}\text { Sociedad de Propagación Marcial y de las Artes } \\
\text { Nacionales de Shanghái }\end{array}$ & Shang Hai Yang Wu Guo Shu Hui & 上海扬武国术会 \\
\hline $\begin{array}{l}\text { Sociedad Experimental de Salud de Jinan Provincia } \\
\text { de Shandong }\end{array}$ & $\begin{array}{c}\text { Shan Dong Ji Nan Shi Yan Jian Kang Xue } \\
\text { She }\end{array}$ & 山东济南实验健康学社 \\
\hline Sociedad Moral de Estudios Marciales de Tianjing & Tian Jing Dao De W u Xue She & 天津道德武学社 \\
\hline Técnicas de Bayoneta y Sable Chinos & Zhong Hua Ci Qiang Shu Yu Da Dao Shu & 中華刺槍術與大刀術 \\
\hline Tercera Clase & Bing Deng & 丙等 \\
\hline Tiro con Arco & She Jian & 射箭 \\
\hline Tiro de Balines & Dan Gong & 弹弓 \\
\hline Trabajo Interno & Nei Gong & 内功 \\
\hline Tres Principios de la Gente & San Min Zhu Yi & 三民主義 \\
\hline Unidades Combinadas de Sables/Cuchillos Largos & Jian Yong Da Dao & 兼用大刀 \\
\hline Valiente & Yong Shi & 勇士 \\
\hline Valor & Quan Yong & 拳勇 \\
\hline Virtud Marcial & Wu De & 武德 \\
\hline Volumen Mensual de Artes Nacionales & Guo Shu Yue Kan (Tian Jin) & 国术月刊/天津 \\
\hline Voz del Arte Nacional & Guo Shu Sheng & 国术声 \\
\hline
\end{tabular}




\begin{tabular}{|c|c|}
\hline \multicolumn{2}{|c|}{ LISTA DE N OMBRES } \\
\hline Pinyin & Caracteres Chinos \\
\hline Jin Jing Zhong & 金警鐘 \\
\hline Li Q in Min & 李親民 \\
\hline Liu Fu Min & 刘福民 \\
\hline Ma Liang & 馬良 \\
\hline Qi Jiguang & 戚繼光 \\
\hline Sun Yat Sen (Sun Yi Xiang) & 孫逸仙 \\
\hline Xie Qiang Gong & 谢强公 \\
\hline Zheng Tian Shi & 郑天轼 \\
\hline Zhuang Nai Gang & 庄乃港 \\
\hline
\end{tabular}

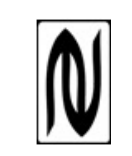

